\title{
Viscous-fingering mechanisms under a peeling elastic sheet
}

\author{
Gunnar G. Peng ${ }^{1} \dagger$, John R. Lister ${ }^{1}$ \\ ${ }^{1}$ Department of Applied Mathematics and Theoretical Physics, University of Cambridge, \\ Wilberforce Road, Cambridge, CB3 0WA, UK
}

(Received xx; revised xx; accepted xx)

We study the mechanisms affecting the viscous-fingering instability in an elastic-walled Hele-Shaw cell by considering the stability of steady states of unidirectional peeling-bypulling and peeling-by-bending. We demonstrate that the elasticity of the wall influences the steady base state but has a negligible direct effect on the behaviour of linear perturbations, which thus behave like in the printer's instability with rigid walls. Moreover, the geometry of the cell can be very well approximated as a triangular wedge in the stability analysis. We identify four distinct mechanisms - surface tension acting on the horizontal and the vertical interfacial curvatures, kinematic compression in the longitudinal base flow, and the films deposited on the cell walls - that each contribute to stabilizing the system. The vertical curvature is the dominant stabilizing mechanism for small capillary numbers, but all four mechanisms have a significant effect in a large region of parameter space.

\section{Introduction}

We consider an elastic sheet that is adhered to a rigid surface by a thin layer of viscous liquid and is being pulled or bent upward at one end, causing the sheet to peel away from the rigid base in a steady motion, as shown schematically in figure 1. McEwan \& Taylor (1966) studied this problem for the case when an applied tension is the only elastic force and found how the peeling velocity $U$ depends on the tension $T$, the slope $\alpha$ at which the sheet is being pulled, the initial height $h_{0}$ of the liquid layer, the viscosity $\mu$ of the liquid and the surface tension $\gamma$ acting on the interface between the liquid and the air drawn in from the lifted end (which was open to the atmosphere). They noted in their experiments that the advancing air-liquid interface under the elastic sheet would sometimes be unstable and develop finger-like undulations, but did not study the instability theoretically.

The instability observed by McEwan \& Taylor (1966) is a version of the classical viscous-fingering instability in a rigid, parallel-walled Hele-Shaw cell (e.g. Saffman \& Taylor 1958; Chuoke et al. 1959), or in a porous medium (e.g. Hill 1952; Homsy 1987), where the advancing interface between an intruding less viscous fluid (in this case air) and the more viscous fluid being displaced (in this case the liquid) develops fingers. The viscosity difference between the fluids provides the driving mechanism of the instability, because near any part of the interface that is perturbed to be in advance of the mean interfacial position, there is less viscous resistance to further advance of the interface, and the perturbation grows. This growth is resisted by surface tension, which acts to straighten out the undulating interface.

More recently, Pihler-Puzović et al. (2012, 2013, 2014) studied the viscous-fingering

$\dagger$ Email address for correspondence: G.G.Peng@damtp.cam.ac.uk 
Slope $\alpha$ or curvature $\kappa$

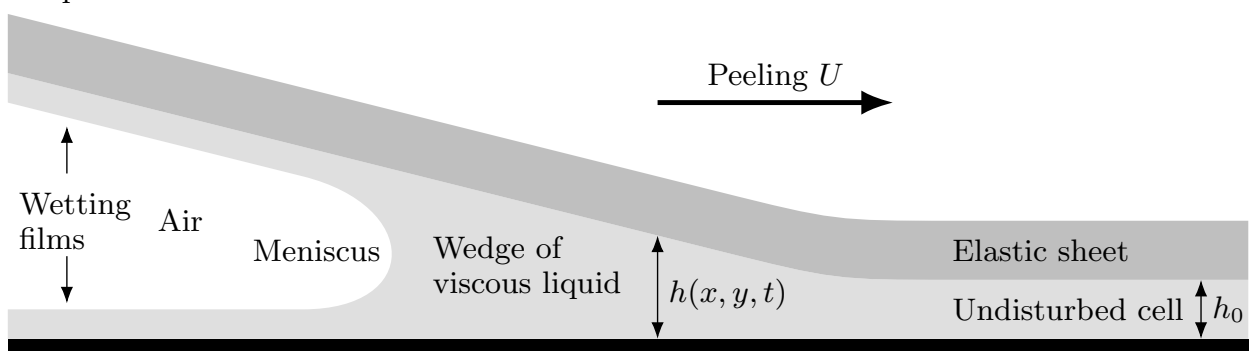

Rigid base

FIgURE 1. Schematic figure of a peeling elastic sheet (under tension $T$ or with bending stiffness $B$ ), which is initially adhered to a rigid base by a liquid layer of undisturbed thickness $h_{0}$, viscosity $\mu$ and surface tension $\gamma$. Peeling proceeds at a speed $U$ due to pulling the left-hand end of the sheet at a slope $\alpha$ or bending it with a curvature $\kappa$.

instability in an elastic-walled Hele-Shaw cell, where air is injected from below into the narrow liquid-filled gap between a rigid base and an overlying elastic sheet. The injected air forms a pancake-shaped bubble which lifts the sheet, causing it to peel away from the base as the bubble grows. Although plenty of data on the instability has been collected from experiments and numerical simulations, so far no conclusive explanation has been given for the observations of Pihler-Puzovic et al. (2012) that the viscousfingering instability in the elastic-walled Hele-Shaw cell is significantly weaker than in a rigid-walled cell, in that much larger injection rates are required to trigger the instability.

Lister et al. (2013), Pihler-Puzović et al. (2015) and Peng et al. (2015) studied the axisymmetric base state of the elastic-walled system in the absence of instability. Their models used the Föppl-von-Kármán equations for the elastic sheet, which include the effects both of tension induced by the deflection of the sheet and of bending forces. They identified two opposite asymptotic limits, in which the peeling is driven purely by tension forces (as in McEwan \& Taylor (1966)) or purely by bending forces, which they called "peeling-by-pulling" and "peeling-by-bending", respectively, and discussed the conditions for when each limit is valid. These peeling processes have also been studied in the case with a single fluid filling the gap under the sheet, and hence no possibility of viscousfingering instability, by e.g. Hosoi \& Mahadevan (2004) and Hewitt et al. (2015).

For bending, the bending stiffness $B$ of the sheet and the applied curvature $\kappa$ (or equivalently the bending moment $M=B \kappa$ ) play an analogous role to the tension $T$ and slope $\alpha$ for pulling. In this paper, we study the viscous-fingering instability for each of these asymptotic peeling solutions, as a first step towards understanding the instability of the full system.

Other examples of systems with peeling motion that is susceptible to viscous fingering include the injection of air into a rectangular channel covered by an elastic sheet (Ducloué et al. 2017) and the motion of an air-filled ruck down an incline (Balmforth et al. 2014).

The peeling motion results in a wedge of liquid accumulating ahead of the air-liquid interface (figure 1), and hence the air is advancing into a converging region. This system has a rigid analogue, in the form of a roll-coating process where liquid is drawn from a bath into the narrow gap between two counter-rotating rollers and separates into two films coating the rollers. In the frame of reference moving with the nearly parallel roller surfaces, the point of separation is the tip of a tongue of air that is continually advancing into a liquid-filled Hele-Shaw cell with converging rigid walls. In this form, 
the viscous-fingering instability is called the printer's instability, and was thoroughly analysed together with many other coating flows by Pearson (1960).

For parallel walls, the vertical curvature of the air-liquid interface (i.e. the curvature seen in a side-on view like figure 1) gives rise to a constant pressure drop across the meniscus that does not have any dynamical effect. However, as highlighted by e.g. Ruschak (1985) and Al-Housseiny et al. (2012), in a cell with converging walls, a finger that protrudes farther into the cell experiences a narrower gap and hence has a larger vertical curvature at the tip. The increased effect of surface tension then provides a restoring force on the finger. This is the so-called "taper" mechanism, which has a stabilizing effect. Al-Housseiny et al. (2013) hypothesized that the instability in the elastic-walled Hele-Shaw cell studied by Pihler-Puzović et al. (2012) can be analysed as a viscous-fingering instability in a rigid tapered cell (whose shape changes slowly with time), and that taper is the dominant mechanism that stabilizes the system, but they did not provide any supporting evidence for this claim.

In this paper, we perform a full linear stability analysis of the steady translating solutions for peeling-by-pulling and peeling-by-bending, in order to determine how the elasticity of the sheet affects the viscous-fingering instability. We model the system using an extension of the lubrication model described by Peng et al. (2015) and derive the governing equations for the linear perturbations in $\S 2$. Our numerical results, including a plot showing the stable and unstable regions of the parameter space, are presented in $\S 3$. In $\S 4$, we show that perturbations to the height of the elastic sheet are negligible, and introduce a "rigid-lid" approximation, which reduces the instability to a variation of the printer's instability and allows us to make significant analytical progress. The central result of this section is an analytical expression for the growth rate which allows us to clearly distinguish which physical mechanisms control the stability of the system. A brief discussion is given in $\S 5$.

\section{Problem setup}

We consider inviscid gas displacing fluid of viscosity $\mu$ in the narrow gap between a horizontal rigid base at $z=0$ and an overlying elastic sheet at $z=h(x, y, t)$, as shown in figure 1 , where $(x, y, z)$ is the position vector and $t$ is time. The sheet is being lifted by pulling or bending at the left-hand end, causing it to peel away from the rigid base in a wave travelling to the right (which we take to be the direction of increasing $x$ ). The gas and liquid are separated by a meniscus at $x=R(y, t)$, which advances as the peeling progresses. The liquid region $x>R(y, t)$ is thus completely filled with liquid, while the gas region $x<R(y, t)$ contains a central tongue of gas bounded by two films of liquid coating the upper and lower surfaces.

We investigate whether variations to the meniscus position $R$ in the spanwise $y$ direction, and to the height profile $h$, grow due to instability, by considering linear perturbations to a $y$-independent base-state solution that is steady in a frame of reference moving at a fixed speed $U$ to the right.

\subsection{Governing equations}

Our physical model is similar to the one investigated by Peng et al. (2015), who discuss several of the assumptions made below in more detail. The horizontal length scales of the system are assumed to be much longer than the vertical length scales, so that vertically integrated quantities can be employed which are functions of the horizontal position $\boldsymbol{x}=(x, y)$ and time $t$ only. We use $\boldsymbol{\nabla}=\left(\partial_{x}, \partial_{y}\right)$ to denote the horizontal gradient 
operator, a prime to denote the longitudinal derivative $\partial_{x}$, in particular, and a dot to denote a time derivative.

The elastic sheet is modelled either as a membrane under isotropic tension or as an elastic beam, which we refer to as the "peeling-by-pulling" and "peeling-by-bending" cases, respectively, following Lister et al. (2013). (These are two limiting cases of the Föppl-von-Kármán equations which describe a linearly elastic sheet with small strain and small slope, as discussed in more detail by e.g. Lister et al. (2013) and Peng et al. (2015).) Thus, the net upward pressure $p(x, y, t)$ on the sheet is related to its deflection $h-h_{0}$ by either

$$
p=-T \nabla^{2} h \quad \text { (pulling) or } \quad p=B \nabla^{4} h \quad \text { (bending), }
$$

where $T$ is the isotropic tension in the sheet or $B$ is its bending stiffness (which is related to the Young's modulus $E$, thickness $d$ and Poisson's ratio $\nu$ by $B=E d^{3} / 12\left(1-\nu^{2}\right)$ ). (We investigate the non-isotropic case where the spanwise component of tension is reduced towards zero in Appendix C.)

We neglect the effects of gravity on both the sheet and the fluid, and measure all pressures relative to atmospheric pressure. In the liquid region ahead of the meniscus, lubrication theory applies and hence the pressure is vertically uniform to leading order and equal to $p$. Gradients in this pressure drive a flow with a parabolic Poiseuille profile, and hence the depth-averaged velocity and the time evolution of the height profile are given by the lubrication equations (Reynolds 1886)

$$
\boldsymbol{u}=(u, v)=-\frac{h^{2}}{12 \mu} \nabla p, \quad \dot{h}=-\boldsymbol{\nabla} \cdot(h \boldsymbol{u})=\boldsymbol{\nabla} \cdot\left(\frac{h^{3}}{12 \mu} \nabla p\right) \quad \text { in } x>R(y, t) .(2.2 a, b)
$$

(It can be shown that horizontal motion of the sheet due to the viscous shear stress is negligible compared to the motion of the fluid.)

In the gas region behind the meniscus, the pressure in the gas (relative to atmospheric) is assumed to be zero. Physically, this could be due to the cell being open to the atmosphere at the left end. Alternatively, if the region under consideration forms part of a larger-scale system such as an elastic-walled Hele-Shaw cell with gas injection (Lister et al. 2013), then the separation of length scales implies that the pressure in the gas is negligible at leading order. The top and bottom surfaces are coated by thin films of liquid, but these films have negligible vertical variation in pressure and negligible pressure jump across the very slightly curved gas-liquid interface. Hence, to a good approximation,

$$
p=0 \quad \text { in } x<R(y, t) .
$$

The liquid and gas regions are connected by a region near the meniscus in which the horizontal length scales of the flow are comparable with the vertical length scales. We assume that the meniscus is nearly perpendicular to the longitudinal $x$-direction, which is the main direction of flow, and that variations in the $y$-direction occur on a length scale much larger than the vertical scale. Hence, the flow in this region is locally similar to that near the meniscus of a bubble advancing in a Hele-Shaw cell with parallel and rigid walls.

Following Peng et al. (2015), we approximate the meniscus region as a sharp interface in the lubrication model by parametrizing the flow in this region using two functions $f_{1}$ and $f_{2}$, which respectively describe the amount of fluid deposited into the thin films behind the meniscus (as seen in figure 1) and the apparent pressure drop across the meniscus region due to capillary and viscous effects. The dependence of these functions on the local instantaneous capillary number $\widetilde{C a}(y, t)=\mu \dot{R} / \gamma$ was taken by Peng et al. 
(2015) to be

$$
f_{1}(\widetilde{C a})=\frac{\widetilde{C a}^{2 / 3}}{0.76+2.16 \widetilde{C a}^{2 / 3}}, \quad f_{2}(\widetilde{C a})=1+\frac{\widetilde{C a}^{2 / 3}}{0.26+1.48 \widetilde{C a}^{2 / 3}}+1.59 \widetilde{C a},
$$

as an approximation to the asymptotic results by Bretherton (1961) and the numerical results by Reinelt \& Saffman (1985). The matching conditions at the meniscus then become

$$
\begin{gathered}
u=\dot{R}\left(1-f_{1}(\mu \dot{R} / \gamma)\right) \quad \text { at } x=R^{+}, \\
{[p]_{-}^{+}=\frac{\pi}{4} \gamma \partial_{y}^{2} R-\frac{2 \gamma f_{2}(\mu \dot{R} / \gamma)}{h} \quad \text { at } x=R,}
\end{gathered}
$$

where $[p]_{-}^{+}$denotes the jump in pressure between the two sides of the interface. In the first equation, which is a kinematic condition, the lubrication velocity $u$ ahead of the meniscus is lower than the meniscus velocity $\dot{R}$ due to the fraction $f_{1}$ of fluid being deposited from the liquid region into the thin films in the gas region rather than being displaced forwards by the meniscus. The second equation, which is a dynamic condition, describes how the pressure drop across the meniscus region is due to the combination of "horizontal surface tension" (i.e. the action of surface tension to straighten out the meniscus region as seen from above) and "vertical surface tension" (i.e. the action of surface tension on the curvature of the meniscus as seen from the side) with a modification factor $f_{2}$ that includes viscous effects.

Although the pressure, which is the second or fourth derivative of $h$ in (2.1), has a discontinuity at the interface, lower derivatives of $h$ do not. Hence, we have the conditions

$$
\left\{\begin{array}{ll}
h \text { and its first derivative are continuous } & \text { (pulling), } \\
h \text { and its first, second and third derivatives are continuous } & \text { (bending), }
\end{array} \text { at } x=R .\right.
$$

Far ahead of the peeling region, the sheet and liquid are undisturbed and hence

$$
h \rightarrow h_{0} \quad \text { as } x \rightarrow \infty .
$$

At the left-hand end, we assume that the elastic sheet is being peeled off either at a fixed slope $\alpha$ (peeling-by-pulling) or with a fixed curvature $\kappa$ (peeling-by-bending),

$$
h^{\prime} \rightarrow-\alpha \quad \text { (pulling), } \quad h^{\prime \prime} \rightarrow \kappa \quad \text { (bending) as } x \rightarrow-\infty,
$$

where primes denote derivatives with respect to $x$. These conditions were shown by Lister et al. (2013) to be the appropriate matching conditions when the peeling region forms the inner boundary-layer region in a matched asymptotic expansion.

From now on, we will, for simplicity, work in a frame of reference moving at constant speed $U$ to the right. The evolution equation $(2.2 b)$ becomes

$$
\dot{h}-U h^{\prime}=-\nabla \cdot(h \boldsymbol{u})=\boldsymbol{\nabla} \cdot\left(\frac{h^{3}}{12 \mu} \nabla p\right) \quad \text { in } x>R(y, t)-U t,
$$

while the remaining equations $(2.1,2.3-2.8)$ remain the same but with the interfacial position $R$ replaced by $R-U t$. (However, we keep $u$ to denote the original velocity in the stationary frame, rather than transforming it into the moving frame.)

\subsection{Base flow}

We first seek a $y$-independent base solution describing steady peeling at the given speed $U$. Due to translational invariance, we can choose the position of the interface to be at 
$x=0$ in the moving coordinate system (i.e. $x=U t$ in the original frame of reference). Using overbars to denote the steady solution, the elastic laws (2.1) become

$$
\bar{p}=-T \bar{h}^{\prime \prime} \quad \text { (pulling) or } \quad \bar{p}=B \bar{h}^{\prime \prime \prime \prime} \quad \text { (bending). }
$$

Combining (2.3), (2.8) and (2.10) yields analytical solutions for the gas region,

$$
\bar{h}=-\alpha x+A_{1} \text { (pulling) } \quad \text { or } \quad \bar{h}=\frac{1}{2} \kappa x^{2}+A_{2} x+A_{3} \text { (bending) } \quad \text { in } x<0,
$$

where $A_{1,2,3}$ are unknown constants. Hence, only the liquid region will need to be solved for numerically.

Integration of the evolution equation (2.9) with $\dot{h}=0$, and use of the far-field condition

$$
\bar{h} \rightarrow h_{0} \quad \text { as } x \rightarrow \infty
$$

from (2.7), yields

$$
\bar{u}=-\frac{\bar{h}^{2}}{12 \mu} \bar{p}^{\prime}=U \frac{\bar{h}-h_{0}}{\bar{h}} \quad \text { in } x>0 .
$$

This equation describes how the leftward flux $\bar{h}(U-\bar{u})$ in the travelling-wave frame is everywhere equal to its value $U h_{0}$ in the far field.

The kinematic and dynamic interfacial conditions (2.5) simplify to

$$
\bar{h}=\frac{h_{0}}{f_{1}}, \quad \bar{p}=-\frac{2 \gamma f_{2}}{\bar{h}} \quad \text { at } x=0^{+} .
$$

In this equation and henceforth, the functions $f_{1}$ and $f_{2}$ and their derivatives are evaluated at the base value $C a=\mu U / \gamma$ unless otherwise stated. The first condition $(2.14 a)$ is readily interpreted as requiring that the flux $U f_{1} \bar{h}$ of fluid being deposited into thin films behind the interface must be equal to the amount $U h_{0}$ of fluid arriving from the far field. If no such films are formed (i.e. $f_{1}=0$ ) then a steady base state would not exist.

Combining the continuity conditions (2.6) with the analytical solutions (2.11) for the gas region yields

$$
\bar{h}^{\prime}=-\alpha \quad \text { (pulling), } \quad \bar{h}^{\prime \prime}=\kappa, \bar{h}^{\prime \prime \prime}=0 \quad \text { (bending) } \quad \text { at } x=0^{+} .
$$

Typically, one would consider $\alpha$ or $\kappa$ as given and seek to determine the unknown peeling speed $U$, but for convenience we will instead assume that $U$ is known (either because it is imposed directly or because the base state has been solved for already), so that two equations in (2.15) simply yield $\alpha$ or $\kappa$ in terms of $U$ and the other parameters of the problem, and only the equation $\bar{h}^{\prime \prime \prime}=0$ for peeling-by-bending is a condition to impose.

There are two independent nondimensional parameters in this problem, which we choose to be the capillary number and the aspect ratio

$$
C a=\frac{\mu U}{\gamma}, \quad \mathcal{A}= \begin{cases}(T / 12 \mu U)^{1 / 3} & \text { (pulling), } \\ \left(B / 12 \mu U h_{0}^{2}\right)^{1 / 5} & \text { (bending). }\end{cases}
$$

The choice of $\mathcal{A}$ is obtained from balancing all terms in the lubrication equation (2.13), assuming that $h \sim h_{0}$ and $x \sim \mathcal{A} h_{0}$. A full nondimensionalization and the resulting governing equations are given in Appendix A, but we retain the use of dimensional quantities in the main text in order to highlight the physical mechanisms more clearly.

Strictly speaking, our governing equations apply to the asymptotic limit $\mathcal{A} \gg 1$, but we will consider all values of $\mathcal{A} \geqslant 1$, with the understanding that for moderate values of $\mathcal{A}$ the results are only qualitatively correct. (Equivalently, we later consider slopes $\alpha_{\mathrm{i}} \leqslant 1$ when in reality $\alpha_{\mathrm{i}} \ll 1$ is necessary for asymptotic accuracy, and in the linear stability 

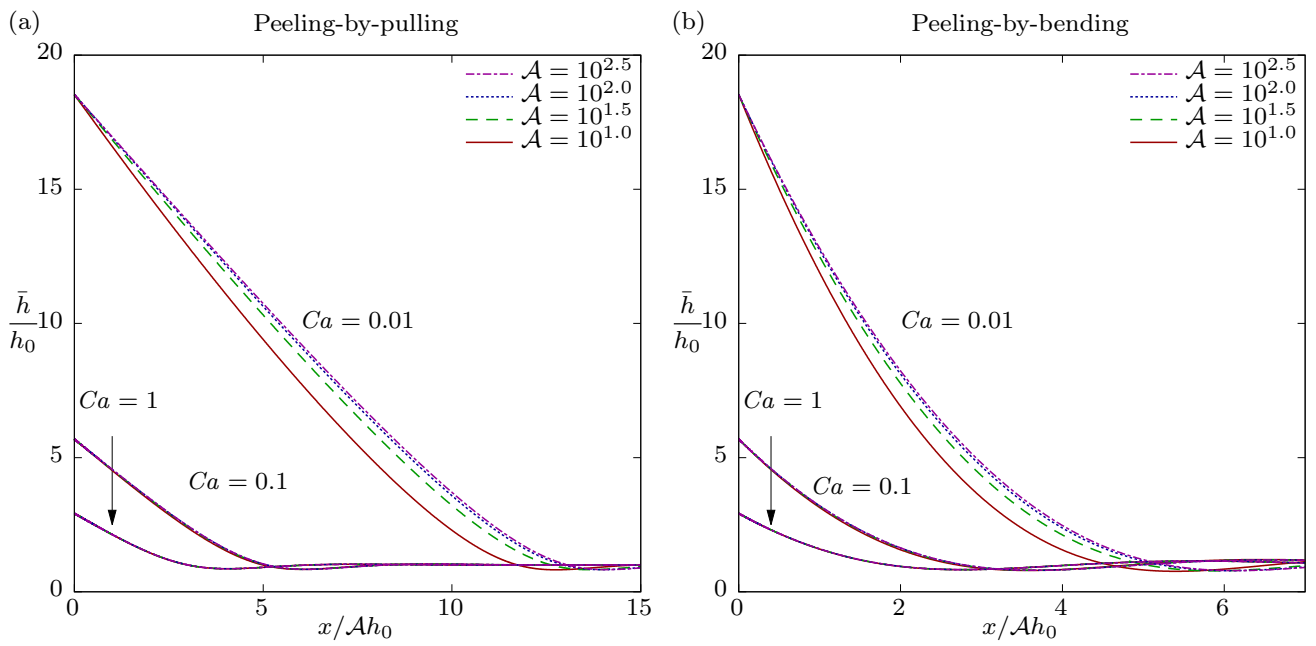

FiguRE 2. Base-state solutions satisfying (2.10)-(2.15) for various values of the capillary number $C a$ and the aspect ratio $\mathcal{A}$ defined in (2.16).

analysis we strictly need $C a \ll 1$ for the main wavelengths to be long compared with the vertical length scale, but we consider all values $C a \leqslant 1$.)

The base-state governing equations (2.10)-(2.15) are straightforward to solve numerically. In fact, both cases have been solved before (McEwan \& Taylor 1966; Balmforth et al. 2014), albeit with different choices of $f_{1}$ and $f_{2}$, which do not change the qualitative behaviour of the system. A few cases are shown in figure 2. The base-state solutions have height $h_{0} / f_{1}$ at the interface, as imposed by $(2.14 a)$ due to conservation of flux. Ahead of the interface, the height decreases towards $h_{0}$, resulting in a wedge-like shape.

Near the tip of the wedge, there are peeling-wave oscillations with length scale $O\left(\mathcal{A} h_{0}\right)$ due to the interplay of the lubrication pressure and the elastic effects (Lister et al. 2013), but these are not a significant effect for the stability calculations here. For peeling-bybending, if $\mathcal{A}$ or $\mathrm{Ca}$ is too small then no steady-state base peeling solution exists (Peng et al. 2015), as can be seen e.g. in figure 5 below. For each given value of $C a$, as $\mathcal{A} \rightarrow \infty$ the solution profile tends to a limiting shape which corresponds to replacing $1 / \mathcal{A}$ by 0 in the pressure-jump condition (A 4), since that is the only occurence of $\mathcal{A}$ in the nondimensional base-state equations (A 2)-(A 4).

As we proceed with the linear stability analysis, two quantities from the base-state solution will be of particular importance. These are the height $h_{\mathrm{i}}$ and slope $\alpha_{\mathrm{i}}$ of the sheet at the interface:

$$
h_{\mathrm{i}}=\bar{h}(0)=\frac{h_{0}}{f_{1}}, \quad \alpha_{\mathrm{i}}=-\bar{h}^{\prime}(0)>0 .
$$

We will also make use of the fact that the longitudinal velocity gradient $\bar{u}^{\prime}$ can be simplified using (2.13) to give

$$
\bar{u}^{\prime}=\frac{U h_{0} \bar{h}^{\prime}}{\bar{h}^{2}}=-\frac{U h_{0} \alpha_{\mathrm{i}}}{h_{\mathrm{i}}^{2}}<0 \quad \text { at } \quad x=0,
$$

which means that the longitudinal flow (in the $x$-direction) is compressive near the interface, due to the flow being stretched vertically as it approaches the meniscus in a widening gap. 


\subsection{Linearized governing equations for small perturbations}

We now derive the equations governing small perturbations to the base states calculated above. Since the base state is independent of $y$ and $t$, we can consider separately perturbations with different wavenumbers $k$ in the $y$-direction and different temporal growth rates $\sigma$ (which we seek to determine as functions of $k$ ). Hence, we decompose each quantity as a sum of the base-state solution and a perturbation with amplitude $\epsilon \ll 1$, real wavenumber $k$ and complex growth rate $\sigma$ :

$$
\begin{aligned}
& h=\bar{h}(x)+\epsilon \operatorname{Re}\left[\hat{h}(x) e^{i k y+\sigma t}\right]+O\left(\epsilon^{2}\right), \quad p=\bar{p}(x)+\epsilon \operatorname{Re}\left[\hat{p}(x) e^{i k y+\sigma t}\right]+O\left(\epsilon^{2}\right), \\
& u=\bar{u}(x)+\epsilon \operatorname{Re}\left[\hat{u}(x) e^{i k y+\sigma t}\right]+O\left(\epsilon^{2}\right), \quad R=U t+\epsilon \operatorname{Re}\left[\hat{R} e^{i k y+\sigma t}\right]+O\left(\epsilon^{2}\right),
\end{aligned}
$$

where Re denotes the real part. We substitute these expressions into (2.1)-(2.9), perform the necessary Taylor expansions, and discard any terms of order $O\left(\epsilon^{2}\right)$ or higher in order to obtain linearized governing equations for the perturbations.

The elastic equations (2.1) become

$$
\hat{p}=T\left(k^{2}-\partial_{x}^{2}\right) \hat{h} \quad \text { (tension), } \quad \hat{p}=B\left(k^{2}-\partial_{x}^{2}\right)^{2} \hat{h} \quad \text { (bending). }
$$

In the gas region, (2.3) becomes simply $\hat{p}=0$, which gives the analytical solution

$$
\hat{h}=A_{4} e^{k x} \quad \text { (pulling), } \quad \hat{h}=\left(A_{5}+A_{6} x\right) e^{k x} \quad \text { (bending) } \quad \text { in } x<0,
$$

where $A_{4,5,6}$ are as yet unknown constants. (We have imposed the condition that $\hat{h} \rightarrow 0$ as $x \rightarrow-\infty$ in order to exclude exponentially growing solutions and thus satisfy (2.8).) The conditions (2.6) for continuity of $h$ and its derivatives at the interface $x=0$ take the form

$$
\begin{array}{ll}
{\left[\hat{h}+\bar{h}^{\prime} \hat{R}\right]_{-}^{+}=\left[\hat{h}^{\prime}+\bar{h}^{\prime \prime} \hat{R}\right]_{-}^{+}=0} & \text { (pulling) } \\
{\left[\hat{h}+\bar{h}^{\prime} \hat{R}\right]_{-}^{+}=\left[\hat{h}^{\prime}+\bar{h}^{\prime \prime} \hat{R}\right]_{-}^{+}=\left[\hat{h}^{\prime \prime}+\bar{h}^{\prime \prime \prime} \hat{R}\right]_{-}^{+}=\left[\hat{h}^{\prime \prime \prime}+\bar{h}^{\prime \prime \prime \prime} \hat{R}\right]_{-}^{+}=0} & \text { (bending), }
\end{array}
$$

and can be combined with $(2.10),(2.14 b)$ and (2.20) to yield the conditions

$$
\begin{array}{lll}
\hat{h}^{\prime}-k \hat{h}+\bar{h}^{\prime \prime} \hat{R}=0 & \text { at } x=0^{+} & \text {(pulling), } \\
\hat{h}^{\prime \prime}-2 k \hat{h}^{\prime}+k^{2} \hat{h}=\hat{h}^{\prime \prime \prime}-2 k \hat{h}^{\prime \prime}+k^{2} \hat{h}^{\prime}+\bar{h}^{\prime \prime \prime \prime} \hat{R}=0 & \text { at } x=0^{+} & \text {(bending), }
\end{array}
$$

on the perturbations in the liquid region. At the opposite end of the liquid region, the far-field condition (2.7) linearizes to

$$
\hat{h} \rightarrow 0 \text { as } x \rightarrow \infty .
$$

In the liquid region itself, the lubrication equations in the travelling-wave frame, $(2.2 a)$ and (2.9), become

$$
\hat{u}=-\frac{\bar{h}^{2}}{12 \mu} \hat{p}^{\prime}-\frac{2 \bar{h} \bar{p}^{\prime}}{12 \mu} \hat{h}, \quad \sigma \hat{h}=\left(\frac{\bar{h}^{3}}{12 \mu} \hat{p}^{\prime}+\frac{3 \bar{h}^{2} \bar{p}^{\prime}}{12 \mu} \hat{h}+U \hat{h}\right)^{\prime}-k^{2} \frac{\bar{h}^{3}}{12 \mu} \hat{p} \quad \text { in } x>0 .
$$

At the interface, the linearized kinematic condition from $(2.5 a)$ is

$$
\hat{u}+\bar{u}^{\prime} \hat{R}=U\left(1-f_{1}-f_{1}^{\prime} C a\right) \sigma \hat{R} \quad \text { at } x=0^{+} .
$$

On the right-hand side, the term $f_{1}$ describes the deposited liquid films, while the term $f_{1}^{\prime} C a$ captures their variation due to the interfacial velocity being perturbed. However, the sum of these two terms is always less than 1 , so the parenthesis remains positive in all cases. 
On the left-hand side of $(2.25 a)$, the first term represents the perturbation flow, which advects the interface and is the main cause of finger growth or decay. The second term describes how longitudinal variations in the base-flow velocity cause the tips of invading air fingers to be advected by a different velocity compared with the base of the fingers. Since $\bar{u}^{\prime}(0)<0(2.17 b)$, we call this effect "kinematic compression", and it helps stabilize the system by advecting the curved interface back towards its mean position.

The linearized dynamic condition is

$$
\hat{p}+\bar{p}^{\prime} \hat{R}=-\frac{\pi}{4} \gamma k^{2} \hat{R}+\frac{2 \gamma f_{2}}{\bar{h}^{2}}\left(\hat{h}+\bar{h}^{\prime} \hat{R}\right)-\frac{2 \gamma f_{2}^{\prime} C a}{\bar{h}} \frac{\sigma \hat{R}}{U} \quad \text { at } x=0^{+},
$$

and we interpret it as quantifying the perturbation pressure $\hat{p}$ at the interface (the first term on the left-hand side) in response to various physical effects. The second term on the left-hand side describes how the lack of viscous pressure drop in the air fingers (compared with in the liquid between the fingers) tends to lead to the pressure in the liquid being larger ahead of the fingers than between the fingers. This is the fundamental driving mechanism of the viscous-fingering instability. In the absence of other effects, it would drive a spanwise flow away from the finger tips and cause them to grow.

On the right-hand side of $(2.25 b)$, the first term describes the stabilizing effect of horizontal surface tension. The middle pair of terms encapsulate the effects of vertical surface tension (as modified by the viscous effects captured by $f_{2}$ ) which cause the liquid pressure ahead of the interface to decrease due to the increase in vertical curvature when the cell height at the interface decreases. The first of this pair of terms describes deflection of the sheet and the second describes the "taper mechanism" (Al-Housseiny et al. 2012). Finally, the last term on the right-hand side of (2.25b) describes the dependence of the dynamic condition $(2.5 b)$ on the meniscus speed via $f_{2}$.

For each value of the wavenumber $k$, equations (2.19) and (2.22)-(2.25) form an eigenvalue problem for the infinite-dimensional eigenvector $(\hat{R}, \hat{h}(x))$ and the associated eigenvalue $\sigma$. We are interested in the dominant eigenmode, i.e. the one whose growth rate has the largest real part and hence grows fastest or decays slowest. Henceforth, we only discuss the dominant solution, and thus consider $\sigma$ as a function of the wavenumber $k$ (for a given base state). If the real part of $\sigma$ is positive, then the dominant eigenmode is growing and the system is linearly unstable. If the real part is negative, then the system is linearly stable to all perturbations with this wavenumber. If the system is linearly stable to perturbations with all wavenumbers $k$, then it is linearly stable.

\section{Linear stability analysis}

\subsection{Review of classical case - rigid parallel cell}

We briefly review the classical case of viscous fingering, where the upper boundary is a rigid horizontal plate. In this case, we have a fixed uniform height $\bar{h}=h_{0}$ with $\hat{h}=0$, instead of the elastic equations (2.1). It follows from flux conservation (2.2b) that $\bar{u}=-h_{0}^{2} \bar{p}^{\prime} / 12 \mu$ is uniform, and the interfacial conditions (2.5) then yield the base solution

$$
\bar{u}=U\left(1-f_{1}\right), \quad \bar{p}=-\frac{2 \gamma f_{2}}{h_{\mathrm{i}}}-\frac{12 \mu U}{h_{\mathrm{i}}^{2}}\left(1-f_{1}\right) x \quad \text { in } x>0,
$$

where we have used $h_{\mathrm{i}}=h_{0}$ for consistency with later equations.

The perturbation pressure is found by solving the lubrication equation (2.24b) with $\hat{h}=0$, which now expresses flux conservation, together with the dynamic meniscus 
condition (2.25b) and the far-field decay condition (2.23). This yields

$$
\hat{p}=\hat{R}\left[\frac{12 \mu U}{h_{\mathrm{i}}^{2}}\left(1-f_{1}\right)-\frac{\pi}{4} \gamma k^{2}-\frac{2 \gamma f_{2}^{\prime} C a}{h_{\mathrm{i}}} \frac{\sigma}{U}\right] e^{-k x} \quad \text { in } x>0 .
$$

Substitution into the kinematic condition $(2.25 a)$ yields the growth rate

$$
\sigma=k U \frac{1-f_{1}-\frac{\pi / 4}{12 C a} k^{2} h_{\mathrm{i}}^{2}}{1-f_{1}-f_{1}^{\prime} C a+\frac{2 f_{2}^{\prime} k h_{\mathrm{i}}}{12}} .
$$

We can trace the various parts of (3.3) back to the physical mechanisms discussed in $\S 2.3$. The last two terms in the denominator capture the dependence of the meniscus conditions (2.5) on the speed of the meniscus via $f_{1}$ and $f_{2}$, but they do not affect the stability of the system (i.e. the sign of $\sigma$ ) since the denominator is always positive. The stability is thus determined by the sign of the numerator. The first part $\left(1-f_{1}\right)$ of the numerator describes the viscous effect that drives the instability, while the second part proportional to $k^{2}$ describes the stabilizing effect of horizontal surface tension. If the wavenumber $k$ exceeds a critical wavenumber $k_{\mathrm{c}}=\left[48 C a\left(1-f_{1}\right) / \pi\right]^{1 / 2} / h_{\mathrm{i}}$, then $\sigma<0$ and the perturbation decays; conversely, if $0<k<k_{\mathrm{c}}$ then $\sigma>0$.

We conclude that in a rigid parallel cell there is always a band $0<k<k_{\mathrm{c}}$ of unstable wavenumbers and the system is unstable regardless of the value of $C a$. However, for $C a \ll 1$, corresponding to strong surface tension, we have $k_{\mathrm{c}} \sim \sqrt{C a} / h_{\mathrm{i}}$ so the unstable wavelengths are very long and the maximum growth rate $\sigma \sim \sqrt{C a} U / h_{\mathrm{i}}$ is small.

Based on this analysis, we define viscous-fingering scales for the wavenumber, growthrate and pressure perturbations,

$$
k_{\mathrm{vf}}=\frac{\sqrt{C a}}{h_{\mathrm{i}}}, \quad \sigma_{\mathrm{vf}}=\frac{\sqrt{C a} U}{h_{\mathrm{i}}}, \quad \hat{p}_{\mathrm{vf}}=\frac{12 \mu U}{h_{\mathrm{i}}^{2}} \hat{R} .
$$

We also introduce a scale for the height perturbations

$$
\hat{h}_{\mathrm{vf}}=\frac{\hat{p}_{\mathrm{vf}}}{T k^{2}} \quad \text { (pulling) } \quad \text { or } \quad \hat{h}_{\mathrm{vf}}=\frac{\hat{p}_{\mathrm{vf}}}{B k^{4}} \quad \text { (bending), }
$$

based on a balance in (2.19) with $\hat{p} \sim \hat{p}_{\text {vf }}$. We will use these scales to nondimensionalize the numerical results and to confirm when the instability is of viscous-fingering type.

\subsection{Numerical results}

Returning to the elastic case, we use a numerical method to investigate the behaviour of (2.19)-(2.25) for various values of the capillary number $C a=\mu U / \gamma$ and the peeling aspect ratio $\mathcal{A}=(T / 12 \mu U)^{1 / 3}$ or $\left(B / 12 \mu U h_{0}^{2}\right)^{1 / 5}$. The equations are discretized using a finite-difference scheme and the dominant linear mode is found using a combination of the Arnoldi algorithm (which is used to find the maximal eigenvalue of a linear operator) and the Newton-Raphson method (which is used to refine the result and for numerical continuation to other parameter values) as described in Appendix A.

We begin by focusing on the particular values $C a=1$ and $\mathcal{A}=10$. Figure 3 shows the structure of the dominant mode for three different values of the spanwise wavenumber $k$, and the base-state height profile for comparison. For both peeling-by-pulling and peelingby-bending, the mode with largest wavenumber decays quickly away from the interface, on the length scale $1 / k$, and becomes negligible before it reaches the tip of the liquid wedge. The modes with smaller wavenumber instead vary on the length scale $L_{\mathrm{w}}$ of the wedge, and reach past the wedge tip into the peeling-wave region. For all $k$, the pressure 
(a)

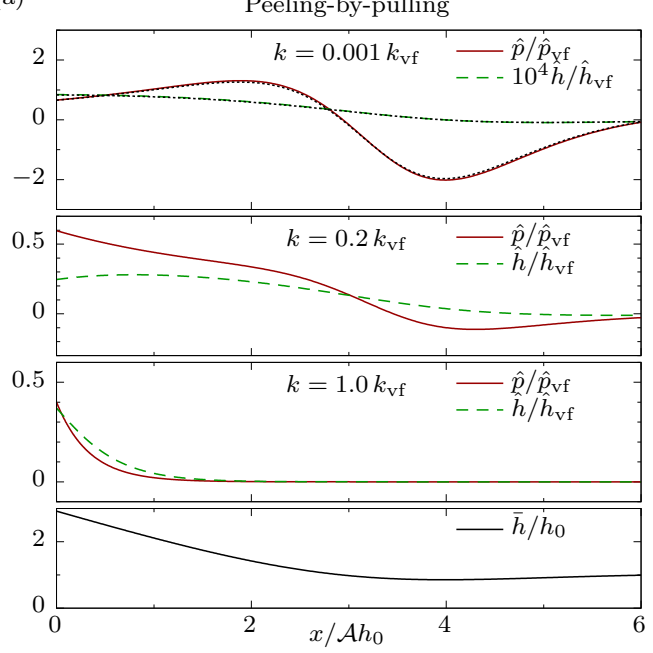

(b)

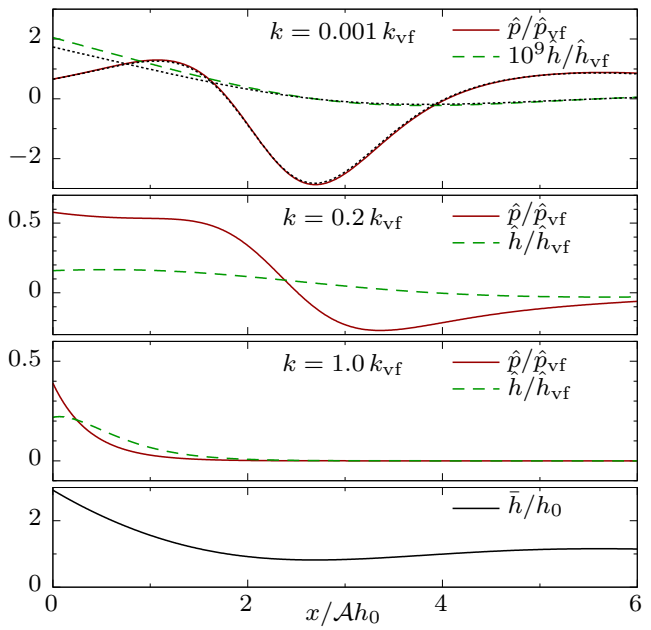

Figure 3. Structure of the pressure and height perturbations of the dominant mode for $C a=1$, $\mathcal{A}=10$, and three values of the spanwise wavenumber $k$, rescaled using the viscous-fingering scales (3.4). In the top row, the translational mode (3.5) is overlaid as dotted curves. Bottom row: Base-state profile showing the extent of the liquid wedge.

(a)

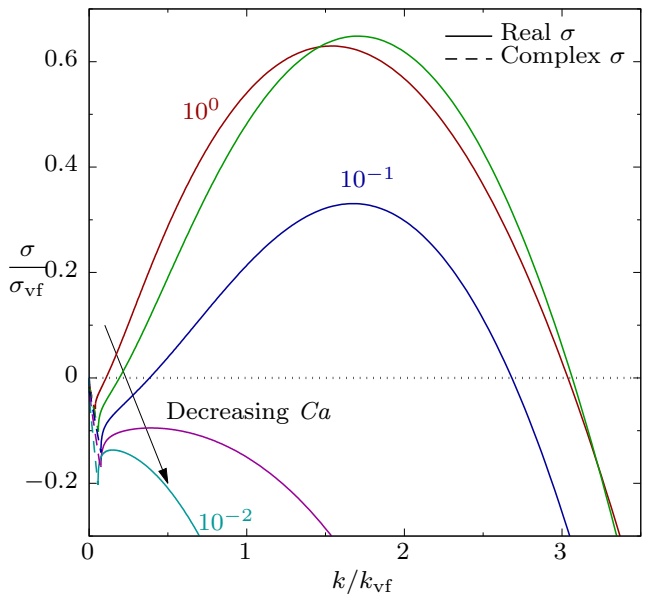

(b)

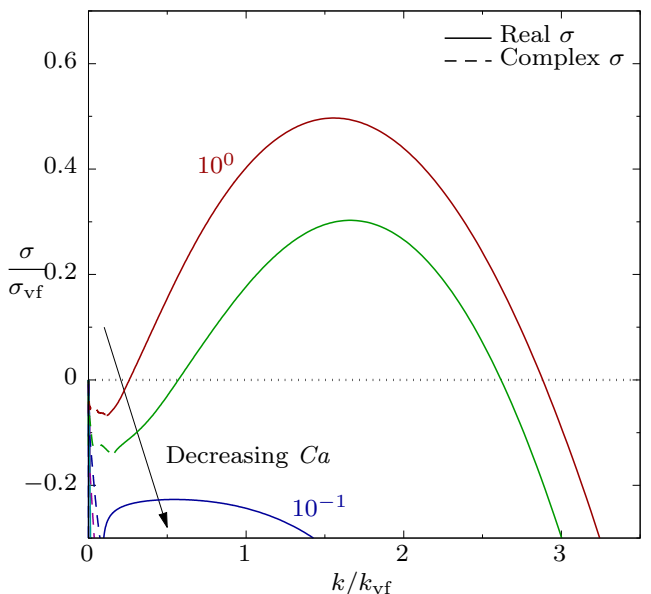

Figure 4. Growth rate $\sigma$ of the dominant mode for each wavenumber $k$, for $\mathcal{A}=10$ and $C a=10^{0}, 10^{-0.5}, 10^{-1}, 10^{-1.5}, 10^{-2}$, rescaled using the viscous-fingering scales (3.4). For the dashed parts of the curves at small $k$, the dominant growth rate is complex and only the real part is plotted. The kinks in the curves for small $k$ correspond to different modes being dominant.

perturbation scales like $\hat{p}_{\mathrm{vf}}$, which is to be expected from a balance on the left-hand side of $(2.25 b)$. The height perturbations scale like $\hat{h}_{\mathrm{vf}}$ for large $k$, when all of the terms balance in (2.19), but for small $k$, the $k^{2}$ terms become negligible compared with the $\partial_{x}^{2} \sim 1 / L_{\mathrm{w}}^{2}$ terms and hence $\hat{h} \ll \hat{h}_{\mathrm{vf}}$.

The growth rates $\sigma$ of the dominant mode for various values of $k$ are shown in figure 4. We have rescaled $k$ and $\sigma$ using the viscous-fingering scales $k_{\mathrm{vf}}=\sqrt{C a} / h_{\mathrm{i}}$ and $\sigma_{\mathrm{vf}}=$ $U \sqrt{C a} / h_{\mathrm{i}}$ from $(3.4 a)$. For not too small $C a$, the system has growing modes for a finite 
range of wavenumbers $k$. For $k$ larger than this range, the growth rate is negative due to the stabilizing effects of surface tension, like in the classical rigid case. For $k$ smaller than this range, there are kinks in the curve, which indicates that different modes are dominant for different ranges of $k$, with each kink being a transition from one mode being dominant to another.

Several of these small- $k$ modes have complex $\sigma$ and hence oscillatory behaviour, in which case we plot only the real part of $\sigma$ in the figure. Since all of the oscillating modes are decaying we will not consider them further, and the modes discussed in detail in this paper all have purely real growth rates.

For $k=0$ the dominant perturbation is a translational mode with $\sigma=0$, which can be found analytically by observing that $\bar{h}(x-\epsilon \hat{R})$ is a steady solution of the original equations (in the travelling-wave frame) for any $\epsilon \hat{R}$, so there exists a linear perturbation

$$
\hat{h}(x)=\lim _{\epsilon \rightarrow 0} \frac{\bar{h}(x-\epsilon \hat{R})-\bar{h}(x, t)}{\epsilon}=-\bar{h}^{\prime}(x) \hat{R}, \quad \hat{p}=-\bar{p}^{\prime} \hat{R}, \quad \hat{u}=-\bar{u}^{\prime} \hat{R}
$$

that satisfies the perturbation equations exactly. Indeed, the numerical results for $k=$ $0.001 k_{\mathrm{vf}}$ in figure 3 agree excellently with the profiles (3.5) shown with dotted curves.

For small $k>0$, a long-wavelength expansion can be performed about the translational mode (3.5); see Appendix B for details. In particular, the leading-order corrections to the translation come from the $O(k)$ terms in the interfacial matching conditions $(2.22)$ for the elastic sheet, and result in a negative $O(k)$ correction to $\sigma$. The physical interpretation is that at long wavelengths each longitudinal strip of the elastic sheet is independent of the others and has the steady-peeling structure at leading order. Where the interface is slightly ahead of its mean position, the peeling solution has advanced further and hence the elastic sheet is slightly higher. Elasticity acts on the resultant spanwise corrugation of the sheet in the gas region to impose a reduction in the peeling slope or curvature at the advanced parts of interface, which slows down their further advance. (The classical viscous and capillary effects of $\S 3.1$ only come in at $O\left(k^{2}\right)$.)

Hence, unlike in the classical case $(\S 3.1), \sigma$ is negative for sufficiently small $k$, and hence there is always a lower bound to the range of unstable wavenumbers $k$ (as well as the upper bound provided by stabilizing capillary effects). The unstable range depends on the system parameters, and as $C a$ decreases, corresponding to surface tension becoming stronger, eventually a critical value (which depends on the value of $\mathcal{A}$ ) is reached below which all perturbations decay and hence the system is stable to linear perturbations.

Given a choice of parameters $(C a, \mathcal{A})$, we can calculate the dominant growth rate $\sigma$ for a range of wavenumbers $k$ to find the maximum growth rate $\sigma_{\mathrm{m}}$, attained at the maximizing wavenumber $k_{\mathrm{m}}$. Figure 5 shows how $\sigma_{\mathrm{m}}$ depends on $C a$ and $\mathcal{A}$. In the shaded region (small $C a$ or $\mathcal{A}$ ), the system is stable, and the maximal growth rate (for any parameter value) is $\sigma_{\mathrm{m}}=0$, attained by the translational mode (3.5). In the unshaded region (large $C a$ or $\mathcal{A}$ ), the system is unstable and the figure shows contours of $\sigma_{\mathrm{m}}$ rescaled by the viscous-fingering scaling $(3.4 a)$.

Figure 5 shows that the maximal growth rate $\sigma_{\mathrm{m}}=O\left(\sigma_{\mathrm{vf}}\right)$ in the unstable region (and we have also verified that the associated wavenumber $k_{\mathrm{m}}=O\left(k_{\mathrm{vf}}\right)$ ), which indicates that the instability is of viscous-fingering type. Reducing $C a$ (e.g. by increasing surface tension) stabilizes the system as expected, like in the classical rigid case. We can give the following heuristic argument for why increasing $\mathcal{A}$ has a destabilizing effect. As $\mathcal{A}$ increases, the base peeling solution becomes longer and thinner, reducing the effect of the taper mechanism discussed in $\S 2.3$ and becoming geometrically more similar to the rigid cell. Hence, the mechanisms that help stabilize the elastic system are likely to be 

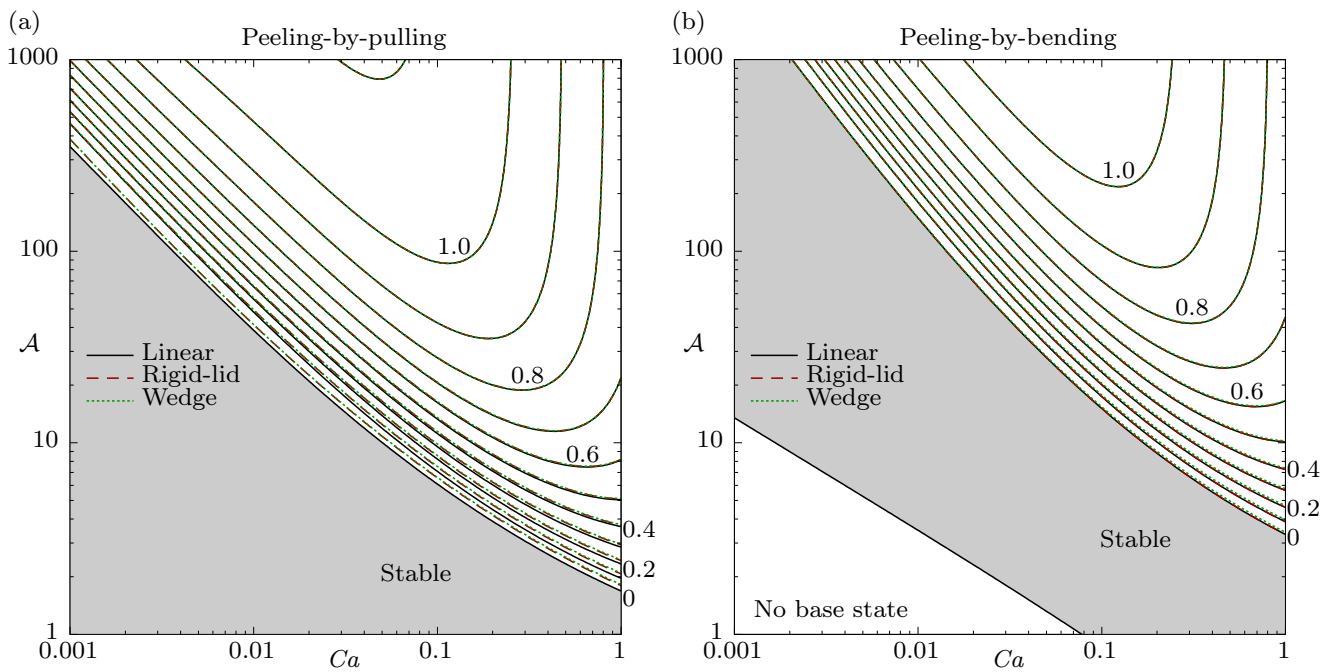

FIGURE 5. Contours of the rescaled maximal growth rate $\sigma / \sigma_{\mathrm{vf}}=\sigma /\left(U \sqrt{C a} / h_{\mathrm{i}}\right)$ as a function of the parameters $C a$ and $\mathcal{A}$. In the shaded region, the system is stable $(\sigma \leqslant 0$ for all $k$ ). In the unshaded region, the contour spacing is 0.1 , with labels indicating the values of some contours. In (b), for sufficiently small $C a$ and $\mathcal{A}$ no steady base-state solution exists. The results from the rigid-lid and wedge approximations from $\S 4$ are shown for comparison.

weaker, making the system more unstable. We will identify these mechanisms and give a more detailed explanation in $\S 4$ below.

\section{Short wavelengths: The rigid-lid approximation}

As we shall see, the horizontal length scale $1 / k$ of the instability is small compared with the length scale of the base-state solution, which we take to be the wedge length $L_{\mathrm{w}} \sim h_{\mathrm{i}} / \alpha_{\mathrm{i}}(2.17 a)$. Hence, the height perturbation $\hat{h} \sim \hat{p} / T k^{2}$ or $\hat{p} / B k^{4}$ generated by the pressure perturbation is small, which allows us to neglect $\hat{h}$ in the main perturbation equations, as if the height of the elastic sheet were unaffected by the flow perturbations. This "rigid-lid" approximation turns out to simplify the problem greatly, and allows significant analytical progress to be made. In particular, it leads to a closed-form expression (4.8) for the growth rate in which different physical mechanisms can be identified.

\subsection{Governing equations for the rigid-lid approximation}

We define the rescaled wavenumber

$$
K=k h_{\mathrm{i}} / \alpha_{\mathrm{i}},
$$

and show in Appendix D.1 that the height perturbations $\hat{h}$ can be neglected both in the lubrication equation (2.24) and in the dynamical interfacial condition $(2.25 b)$ if $K \gg 1$. We will make these simplifications, but allow more moderate values $K \gtrsim 1$ in general.

The lubrication equation (2.24) simplifies to

$$
\hat{u}=-\frac{\bar{h}^{2}}{12 \mu} \hat{p}^{\prime}, \quad 0=\left(\overline{h^{3}} \hat{p}^{\prime}\right)^{\prime}-k^{2} \bar{h}^{3} \hat{p} \quad \text { in } x>0 .
$$

Equation (4.2b) expresses flux conservation in terms of the perturbation pressure $\hat{p}$ but, unlike the classical case $(\S 3.1)$ where $\bar{h}$ is a constant, $\hat{p}$ must be found numerically. However, since (4.2) is linear, homogeneous and second order, and there is a homogeneous 
boundary condition $\hat{p} \rightarrow 0$ as $x \rightarrow \infty$, the solution is determined up to a multiplicative constant. We can calculate a numerical solution $\hat{p}=\hat{p}_{\text {rig }}(x)$ for the arbitrary normalization $\hat{p}_{\text {rig }}(0)=1$, and the actual solution is then given simply by

$$
\hat{p}(x, t)=\hat{p}(0, t) \hat{p}_{\text {rig }}(x) .
$$

In particular, we can express the perturbation velocity $(4.2 a)$ at the interface as

$$
\hat{u}(0, t)=\frac{h_{\mathrm{i}}^{2}}{12 \mu} k Y(C a, \mathcal{A}, k) \hat{p}(0, t), \quad \text { where } \quad Y(C a, \mathcal{A}, k)=-\frac{\hat{p}_{\text {rig }}^{\prime}(0)}{k \hat{p}_{\text {rig }}(0)} .
$$

The quantity $Y$, which we call the "admittance", depends only on the base-state profile $\bar{h}(x)$ and the wavenumber $k$, and captures how the geometry of the cell affects the perturbation flow. For a rigid parallel cell $\left(\bar{h}=h_{0}\right)$, the perturbation pressure profile would be exponential like $(3.2), \hat{p}_{\text {rig }}(x)=\exp (-k x)$, and we would obtain $Y=1$. For the non-parallel peeling geometry, the converging cell restricts the flow and hence a given pressure perturbation results in a weaker perturbation flow, and we find that $Y<1$.

For perturbations that decay rapidly away from the meniscus $(K \gg 1)$, we can use a Taylor expansion of $\bar{h}$ about the meniscus position:

$$
\bar{h}(x) \approx \bar{h}(0)+\bar{h}^{\prime}(0) x=\alpha_{\mathrm{i}} s, \quad \text { where } s=h_{\mathrm{i}} / \alpha_{\mathrm{i}}-x .
$$

We call this the "wedge approximation", as it is equivalent to approximating the basestate profile as being a triangular wedge. Inserting this approximation into (4.2) and solving for $\hat{p}$ with the condition that $\hat{p}$ does not diverge as $s \rightarrow 0$ yields

$$
\hat{p} \propto \frac{I_{1}(k s)}{k s},
$$

where $I_{1}$ is the modified Bessel function of the first kind with order 1 (cf. Grenfell-Shaw \& Woods 2017). This result for $\hat{p}$ gives the admittance as

$$
Y_{w}(K)=\frac{I_{1}^{\prime}(K)}{I_{1}(K)}-\frac{1}{K}, \quad Y_{w}(K) \sim 1-\frac{3}{2 K}+\frac{3}{8 K^{2}} \rightarrow 1 \text { as } K \rightarrow \infty,
$$

where $K=k h_{\mathrm{i}} / \alpha_{\mathrm{i}}$ as before.

Finally, neglecting $\hat{h}$ in the interfacial conditions (2.25), and combining with (4.4), yields the main result for the growth rate,

$$
\sigma=k U \frac{Y\left(1-f_{1}-\frac{\pi / 4}{12 C a} k^{2} h_{\mathrm{i}}^{2}-\frac{2 f_{2}}{12 C a} \alpha_{\mathrm{i}}\right)-\frac{-\bar{u}^{\prime}(0)}{k U}}{1-f_{1}-f_{1}^{\prime} C a+\frac{2 f_{2}^{\prime} Y k h_{\mathrm{i}}}{12}} .
$$

(We have refrained from eliminating $h_{\mathrm{i}}$ and $-\bar{u}^{\prime}(0)$ using $(2.17)$ in order to highlight the physical interpretation of the various terms.)

As in the classical rigid case $(\S 3.1)$, the denominator of (4.8) contains terms which capture the dependence of the meniscus conditions (2.5) on the speed of the meniscus via $f_{1}$ and $f_{2}$, but it remains positive and hence does not affect the stability of the system. In the numerator, we recognize the first three terms as describing the viscous driving force and horizontal surface tension. The fourth term captures the stabilizing effect of the taper mechanism as discussed in $\S 2.3$. These first four terms describe the pressure perturbation generated by a displacement of the interface, and are multiplied by the admittance $Y$ which quantifies how the flow response to the pressure perturbation is reduced by the converging geometry of the cell. Finally, the fifth term captures the effect of kinematic compression, which is stabilizing since $\bar{u}^{\prime}<0$. 
(a)
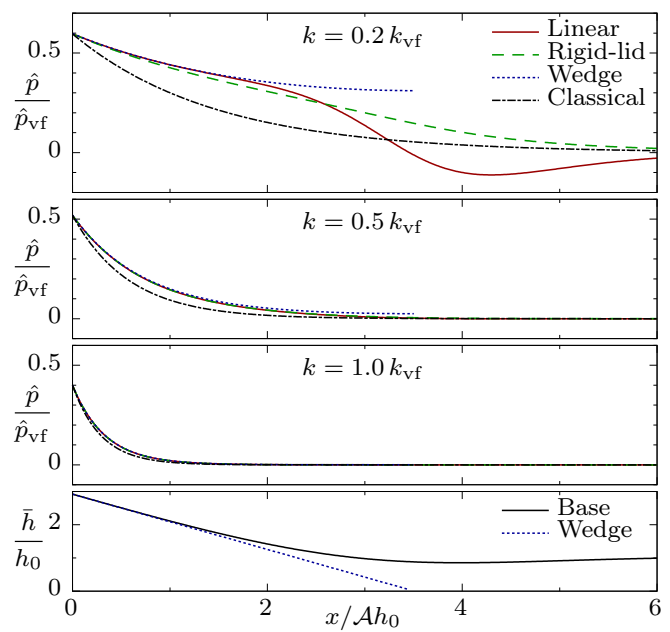

(b)
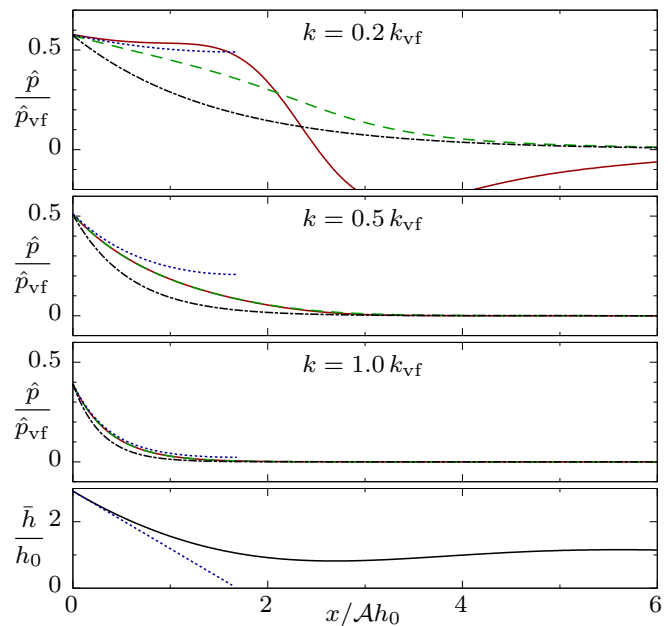

Figure 6. Profiles of the pressure perturbations of the dominant mode for $C a=1, \mathcal{A}=10$ and three values of $k$, rescaled using the viscous-fingering scales (3.4a), comparing results from the full linear calculation and the rigid-lid and wedge approximations (cf. figure 3). The exponential profiles for the classical rigid case are shown rescaled so that they agree with the other results at the interface $x=0$. Bottom row: Base-state height profile and the wedge-approximation profile (4.5).

\subsection{Comparison with full linear calculation}

Figure 6 shows perturbation pressure profiles from the full linear calculation (two of which also shown in figure 3) with the rigid-lid and wedge approximations overlaid. We find that for the largest value of $k$ the approximations are excellent throughout the domain. For smaller values of $k$, the approximations are excellent near the meniscus but do not fare as well towards the tip of the wedge. However, the approximations can still be expected to give a reasonable estimate of the growth rate $\sigma$, since it is determined mainly by the behaviour near the meniscus. For even smaller values of $k$ (not shown), the approximations fail, as is to be expected when the wavelength of the perturbation is greater than the wedge length.

For comparison, the exponential profiles $\hat{p} \propto e^{-k x}$ (with an arbitrary overall factor) for a rigid parallel cell are also shown in figure 6 . The exponential profile agrees with the others only if $k$ is very large, corresponding to the perturbation decaying on such a short length scale that the converging geometry of the cell has no effect. For smaller values of $k$, there is a distinct difference between the profiles, and the rigid-lid and wedge approximations constitute a genuine improvement over treating the cell as approximately parallel.

Figure 7 shows the growth rate $\sigma$ as a function of wavenumber $k$ for $\mathcal{A}=10$ and various values of $C a$, similarly to figure 4 . We find that the rigid-lid and wedge approximations are in excellent agreement with the full linear results over the full range of wavenumbers relevant for the viscous-fingering instability. Only for much smaller $k$, where $\sigma<0$, do they deviate from the full linear results, and predict that $\sigma$ tends to a negative value as $k \rightarrow 0$ when in reality $\sigma \rightarrow 0$. The contour plot of the rescaled maximal growth rate $\sigma_{\mathrm{m}} / \sigma_{\mathrm{vf}}$ shown in figure 5 includes the results from the rigid-lid and wedge approximations, and we observe that the excellent agreement extends to the entire parameter space. We conclude that the rigid-lid and wedge approximations have completely captured the physics of the viscous-fingering instability in the elastic-walled Hele-Shaw cell. 

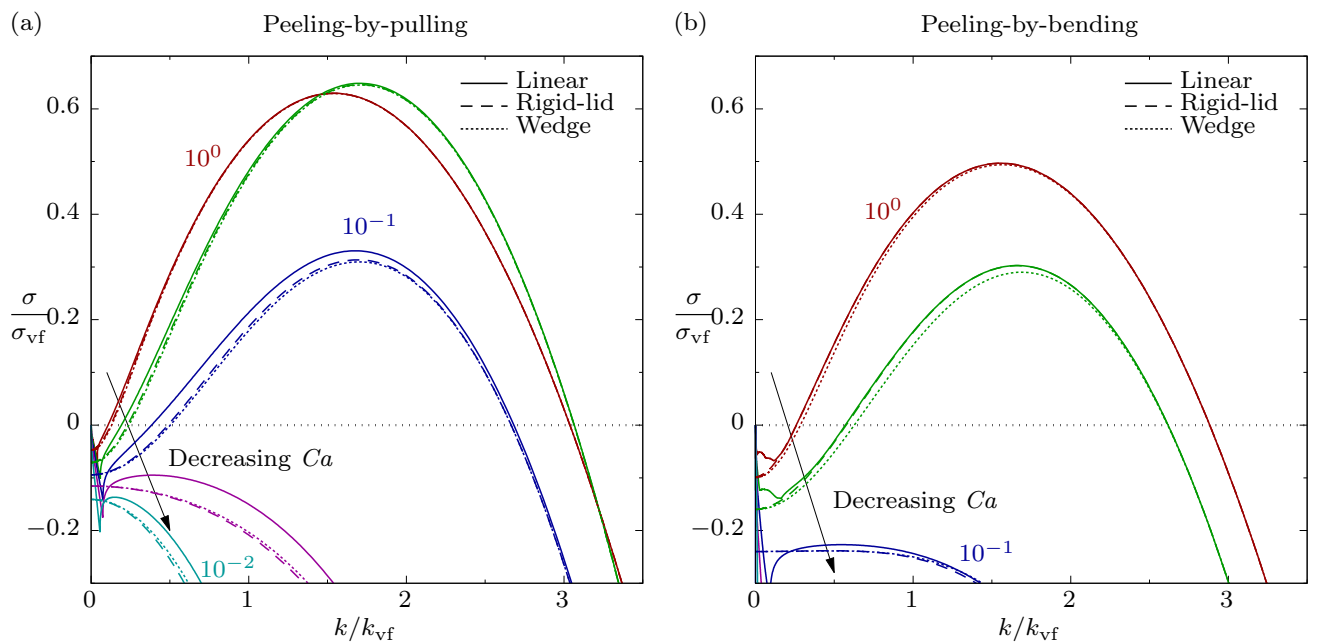

FiguRe 7. Growth rate $\sigma$ of the dominant mode for each wavenumber $k$, for $\mathcal{A}=10$ and $C a=10^{0}, 10^{-0.5}, 10^{-1}, 10^{-1.5}, 10^{-2}$, comparing results from the full linear calculation and the rigid-lid and wedge approximations (cf. figure 4).
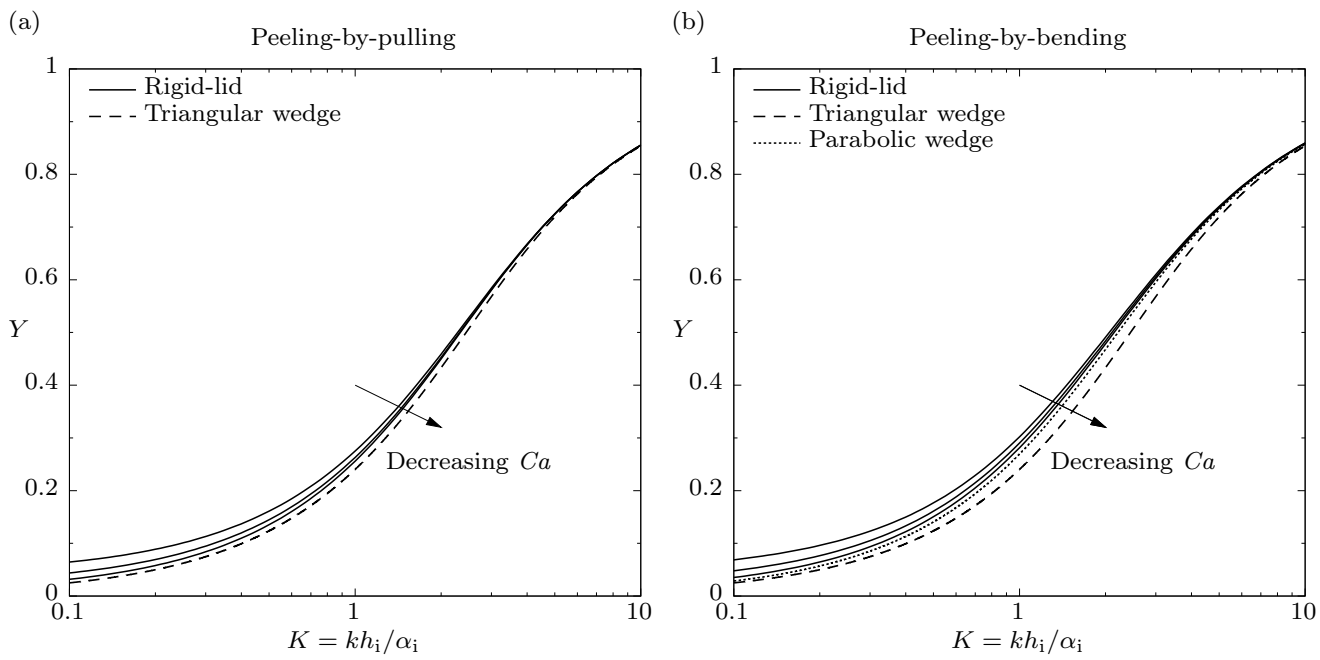

Figure 8. Admittance $Y$ calculated from the rigid-lid approximation for $\mathcal{A}=10$ and $C a=10^{0}, 10^{-0.5}, 10^{-1}$, and from the wedge approximation (4.7). In (b), results for a parabolic wedge (E 1$)$ are also shown.

\subsection{Comparison between rigid-lid and wedge approximations}

The rigid-lid and wedge approximations differ only in the calculation of the admittance $Y$ : the rigid-lid approximation uses the actual base-state profile $\bar{h}(x)$, whereas the wedge approximation goes further and uses a simple triangular wedge profile (4.5) with the same height and slope at the interface.

Figure 8 shows a comparison between the admittance calculated from the rigid-lid approximation (for $\mathcal{A}=10$ and various values of $\mathrm{Ca}$ ) and from the wedge approximation $Y=Y_{w}(K)$ (which is a function of $K$ alone). We find excellent agreement between the two for large $K=k h_{\mathrm{i}} / \alpha_{\mathrm{i}}$ (i.e. $1 / k \ll L_{\mathrm{w}}$ ), as expected, since the wedge approximation 


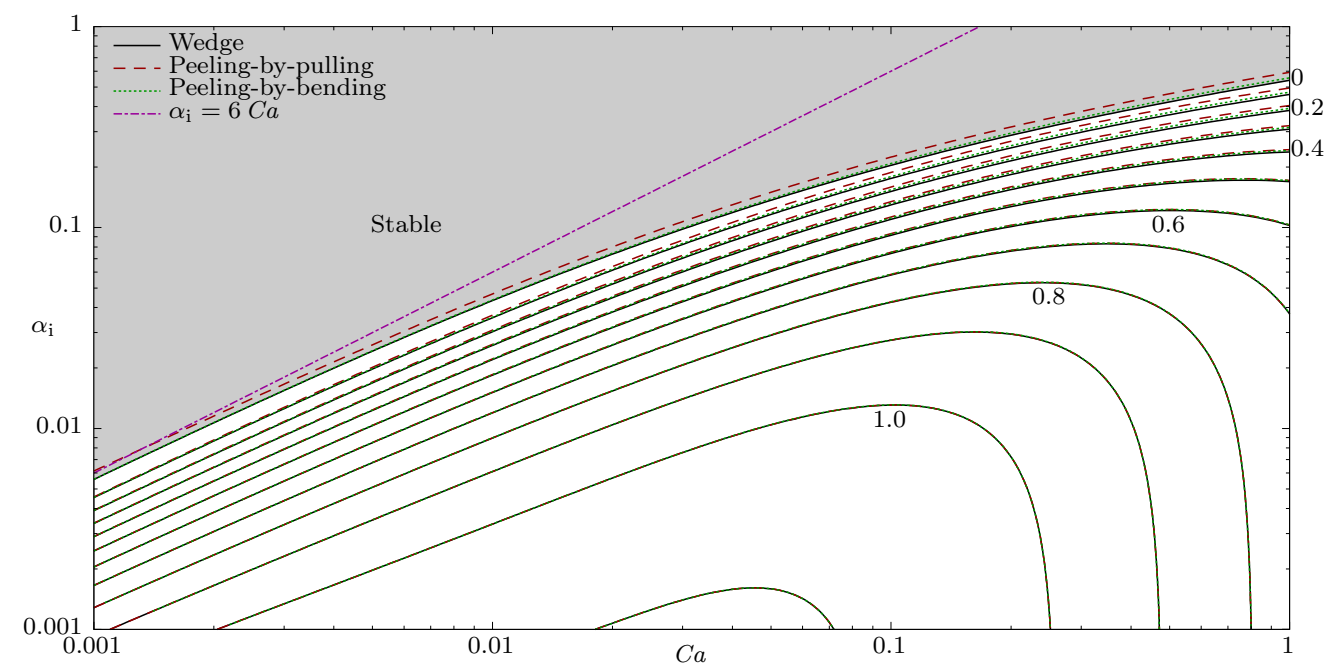

FiguRE 9. Contours of the rescaled maximal growth rate $\sigma_{\mathrm{m}} / \sigma_{\mathrm{vf}}=\sigma_{\mathrm{m}} /\left(U \sqrt{C a} / h_{\mathrm{i}}\right)$ (cf. figure 5 ) as a function of capillary number $C a$ and slope $\alpha_{\mathrm{i}}$ for peeling-by-pulling and peeling-by-bending under the full linear calculation, and for the universal wedge approximation. The stability boundary $\alpha_{\mathrm{i}}=6 C a(4.10)$ for $C a \ll 1$ is also shown.

is derived from a Taylor expansion of the rigid-lid profile. As $K$ decreases towards 1 , the approximations start to differ since $1 / k \sim L_{\mathrm{w}}$. For all $K$, as $C a$ decreases (and the wedge becomes larger), the rigid-lid results approach those for the wedge, which suggests that the base-state profile is becoming more triangular. For peeling-by-pulling, the agreement is excellent. For peeling-by-bending (figure $8 b$ ), slightly better results are obtained by expanding to second order and approximating the wedge as parabolic; see Appendix E for a detailed discussion.

Under the wedge approximation, the expression (4.8) for the growth rate $\sigma$ does not depend directly on $\mathcal{A}$ or the elasticity model for the lid. Instead, these affect the base-state profile and hence the slope $\alpha_{\mathrm{i}}$, which appears in (4.8) explicitly as well as via the admittance for the wedge approximation (4.7) and via the compression term $(2.17 b)$. Apart from an overall dimensional factor $U / h_{\mathrm{i}}$ (or equivalently $U / h_{0}$, since $\left.h_{\mathrm{i}}=h_{0} / f_{1}(C a)\right)$, the growth rate $\sigma$ thus only depends on the capillary number $C a$, the slope $\alpha_{\mathrm{i}}$ at the interface, and the rescaled wavenumber $k h_{\mathrm{i}}$. Maximizing $\sigma$ over all $k h_{\mathrm{i}}$ yields the maximal growth rate $\sigma_{\mathrm{m}}$, which apart from the overall factor $U / h_{\mathrm{i}}$ now only depends on $C a$ and $\alpha_{\mathrm{i}}$.

Figure 9 shows contours of the rescaled growth rate as a function of $C a$ and $\alpha_{\mathrm{i}}$ for the full linear calculations and the wedge approximation. We notice that the results for both peeling-by-pulling and peeling-by-bending collapse excellently onto the universal result obtained from the wedge approximation. This confirms that the instabilities in this system behave like viscous-fingering instabilities in a rigid triangular wedge, and that the aspect ratio $\mathcal{A}$ and the elasticity of the lid only affect the instability via the slope of the lid at the meniscus.

\subsection{Analysis of contributions to stability}

Having verified the rigid-lid and wedge approximations against the full linear calculations, we can now analyse the expression (4.8) for the growth rate to determine the relative importance of the various physical effects. The condition $\sigma<0$ for stability of 

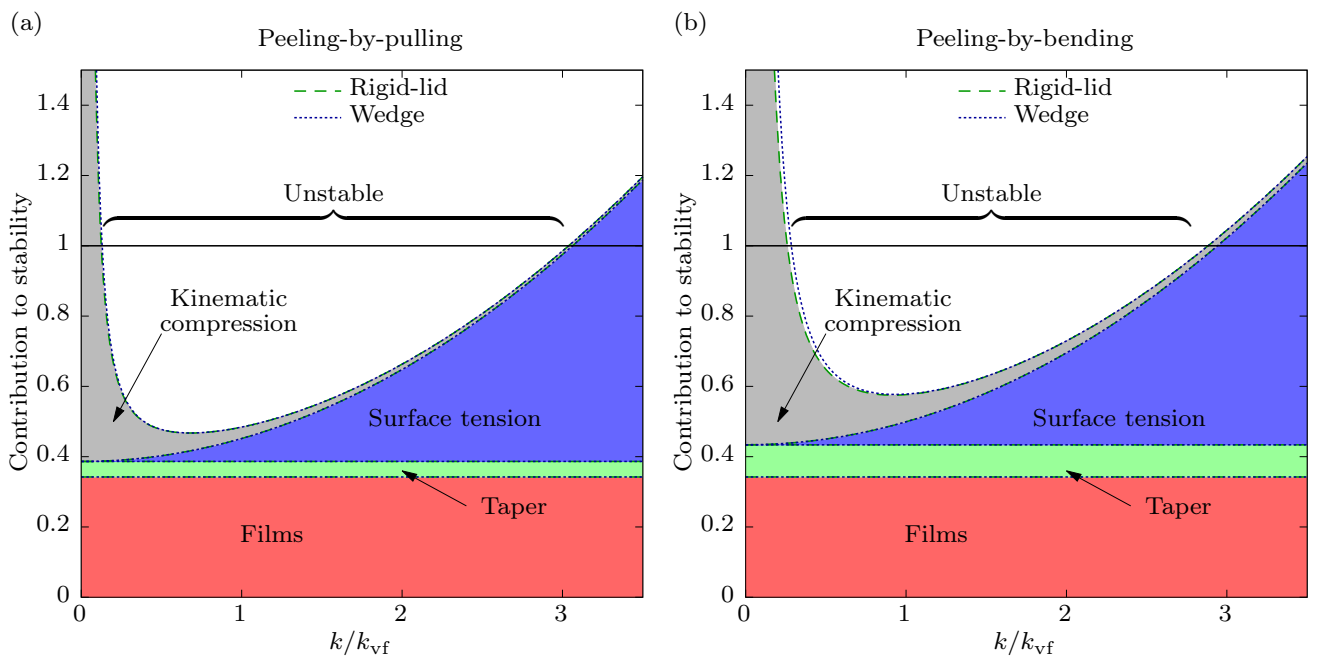

Figure 10. The contribution to stability of the four terms in (4.9), stacked from bottom to top, for $C a=1$ and $\mathcal{A}=10$, as a function of wavenumber $k$, under the rigid-lid and wedge approximations.

the mode with a given wavenumber $k$ is equivalent to the condition

$$
f_{1}+\frac{2 f_{2}}{12 C a} \alpha_{\mathrm{i}}+\frac{\pi / 4}{12 C a} k^{2} h_{\mathrm{i}}^{2}+\frac{-\bar{u}^{\prime}(0)}{k U Y}>1 .
$$

The four terms on the left-hand side are positive, so we interpret them as the "contributions to stability" of four distinct physical mechanisms.

We recognize the first and third terms in (4.9) from the classical rigid case. They represent the effects of wetting films reducing the driving viscous pressure drop and of the horizontal surface tension acting to straighten out the interface. The second term represents the taper mechanism providing a larger resisting pressure drop across the meniscus on fingers penetrating deeper into the converging cell. The fourth term represents kinematic compression in the base flow providing a restoring advection, and this effect is strengthened by the reduction in admittance $Y$ due to the converging cell.

We plot the four contributions to stability in (4.9) using stacked-area graphs to highlight both their sum and the relative importance of each term. For example, figure 10 shows the contributions as a function of the wavenumber $k$ for the case $C a=1$ and $\mathcal{A}=10$. The wavenumbers for which the sum is less than 1 are unstable (cf. figure 7). Since the base state is fixed, the terms representing films and taper have a constant contribution, while the horizontal surface tension stabilizes larger wavenumbers and kinematic compression stabilizes smaller wavenumbers.

The curve of marginal stability, i.e. the stability boundary, is important since its location in figures 5 and 9 determines whether fingering occurs in any given physical system. On the curve, the sum of the four contributions to stability in (4.9) is larger than 1 for all $k$ except the wavenumber of the crucial "marginal mode", for which the sum equals 1 and the growth rate is exactly $\sigma=0$.

For each value of $C a$, we calculate (under both the rigid-lid or wedge approximation, which are in excellent agreement) the marginally stable base state, the associated marginal mode, and the contributions to stability of the various physical mechanisms for this mode. The wavenumber $k$ of the marginal mode, shown in figure $11(a)$, is $O\left(k_{\mathrm{vf}}\right)$ for this range of $C a$, but $k / k_{\mathrm{vf}}$ decreases (very) slowly as $C a \rightarrow 0$. The contributions to 

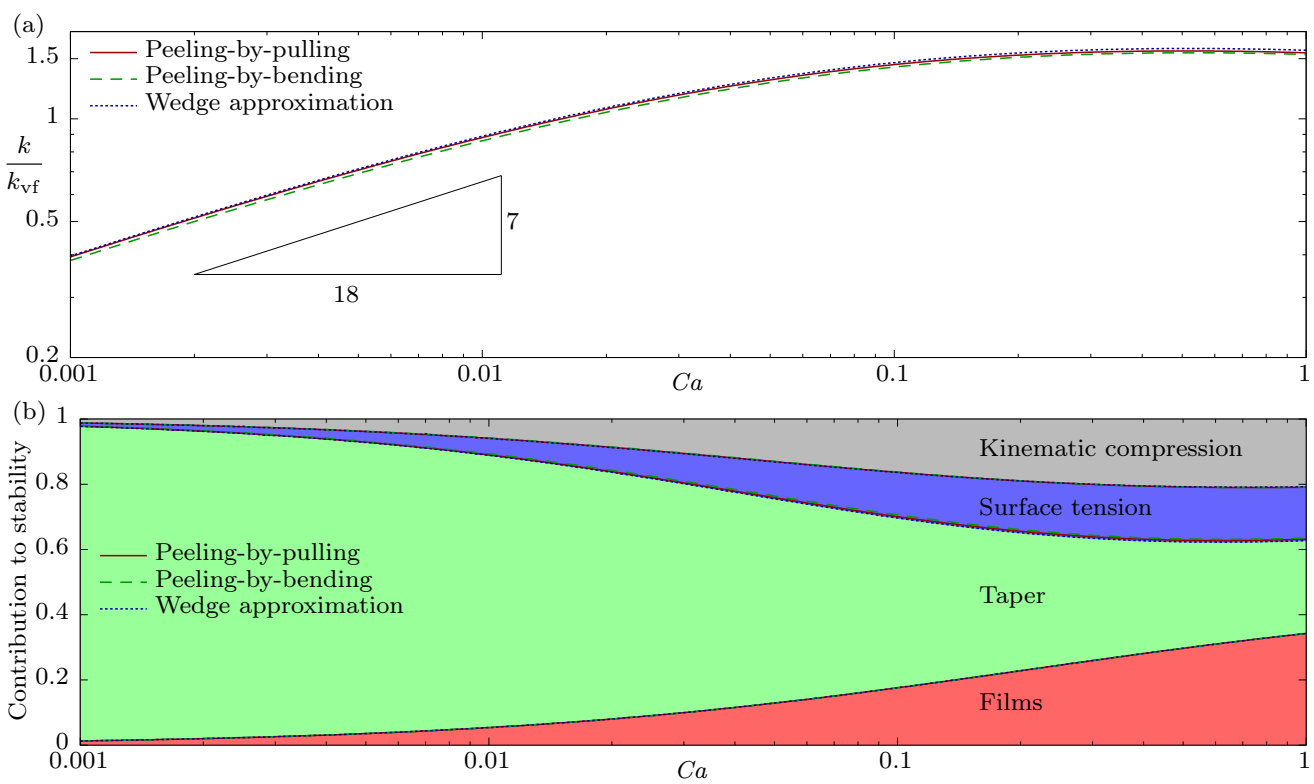

Figure 11. Properties at marginal stability (i.e. the boundary between stable and unstable in parameter space) as functions of $C a$, for peeling-by-pulling and peeling-by-bending using the rigid-lid approximation, and using the wedge approximation. (a) The wavenumber $k$ of the marginal mode, rescaled using the viscous-fingering scale $k_{\mathrm{vf}}=\sqrt{C a} / h_{\mathrm{i}}(3.4 a)$. The power law $k / k_{\mathrm{vf}}=O\left(C a^{7 / 18}\right)$ is predicted by (D $\left.4 b\right)$. (b) The contribution to stability of the four terms in (4.9) for the marginal mode, stacked from bottom to top.

stability (4.9), shown in figure $11(b)$, sum exactly to 1 as expected, and for $C a \gtrsim 0.1$ all four terms are of comparable magnitude. We conclude that all four mechanisms play an important role in stabilizing the system for moderate $C a$, and neglecting any one of them would yield a significantly different prediction for the stability boundary.

In the limit $C a \rightarrow 0$, the contribution to stability of the taper term dominates in figure $11(b)$. Hence, the condition (4.9) for the system to be stable to perturbations of all wavenumbers reduces to

$$
\alpha_{\mathrm{i}}>\frac{12 C a}{2 f_{2}} \approx 6 C a
$$

(cf. Al-Housseiny et al. 2012), which agrees well with the full linear results in figure 11(b). A more detailed analysis of the limit $C a \rightarrow 0$ for the unstable region, given in Appendix D.2, shows that the wavenumber $k_{\mathrm{m}}$ of the mode with maximal growth rate $\sigma_{\mathrm{m}}$ satisfies $k_{\mathrm{m}} \sim k_{\mathrm{vf}}$ away from the stability boundary but decreases to $k_{\mathrm{m}} \sim C a^{7 / 18} k_{\mathrm{vf}}$ (D $4 b)$ on the stability boundary, which is in excellent agreement with the results in figure $11(a)$. The analysis also shows that $K=k_{\mathrm{m}} h_{\mathrm{i}} / \alpha_{\mathrm{i}} \gg 1$, which justifies the use of the rigid-lid approximation, and that the asymptotic corrections (D 6) to the leadingorder result (4.10) are of relative order $O\left(\mathrm{Ca}^{2 / 3}\right)$ (due to the effects of films and the correction to $f_{2}$ ) and $O\left(\mathrm{Ca}^{7 / 9}\right)$ (due to the effects of horizontal surface tension and kinematic compression). 


\section{Discussion}

\subsection{Summary}

We have investigated the linear stability of the peeling of an elastic sheet adhered to a rigid substrate by a layer of viscous liquid. The sheet was modelled as either a membrane under tension $T$ (peeling-by-pulling) or an elastic beam with bending stiffness $B$ (peeling-by-bending).

Using a linear stability analysis, we determined which regions of the two-dimensional parameter space are stable and which are unstable (figures 5 and 9). Comparison of the full analysis with a rigid-lid approximation and a wedge approximation revealed that any growing perturbations are of viscous-fingering type with a sufficiently short length scale that the perturbations to the lid are unimportant and that its base-state profile can be approximated using the linear terms in a Taylor expansion. We have thus demonstrated conclusively that the elasticity of the lid only affects the instability by altering the profile of the base peeling solution, while the linear perturbations to the lid profile are negligibly small. In addition, the only properties of the base-state profile that matter for determining the growth rate of the perturbations are the height $h_{\mathrm{i}}$ and slope $\alpha_{\mathrm{i}}$ at the interface, and so the linear instability behaves like the printer's instability in a rigid triangular wedge.

The rigid-lid and wedge approximations yield an analytical expression (4.8) for the growth rate, allowing the condition $\sigma<0$ for stability to be rewritten in the form (4.9), which requires the sum of four positive terms to be greater than 1 . We interpret these terms as four distinct stabilizing physical mechanisms.

Two mechanisms are present also in the classical rigid case, and represent films deposited behind the meniscus (which reduce the base viscous pressure drop that drives the instability) and horizontal surface tension (which acts to straighten out the interface). The other two mechanisms are only present in a converging cell: the taper mechanism (which provides a larger resisting pressure drop across the meniscus on fingers penetrating deeper into the converging cell) and kinematic compression in the base flow (which advects the interface back towards its mean position). The relative effect of kinematic compression is strengthened by the reduction in admittance $Y$ (which quantifies the strength of the flow response to a destabilizing pressure perturbation) in a the converging cell.

The magnitude of each term in (4.8) quantifies the relative importance of each mechanism in contributing to the stability of the system. The stabilizing effect of films and taper is independent of the wavenumber $k$, while horizontal surface tension suppresses instability for large $k$ and kinematic compression suppresses instability for small $k$. For small $C a$, the taper term dominates, and we asymptotically obtain the stability condition $\alpha_{\mathrm{i}}>6 C a$ (cf. Al-Housseiny et al. 2012). However, for moderate $C a=\mu U / \gamma$, all four terms are important near the stability boundary (figure 11b), and omitting any one of them would yield inaccurate results. This holds for the experiments by McEwan \& Taylor (1966), Pihler-Puzović et al. (2012) and Ducloué et al. (2017), in which the capillary number was typically in the range $0.1 \leqslant C a \leqslant 10$.

\subsection{Some generalizations}

Our results were derived using interfacial conditions (2.5) which are asymptotically accurate in the limit $C a \rightarrow 0$ but only approximate for larger values of $C a$, and which require $k h_{\mathrm{i}} \ll 1$. Nevertheless, we expect our qualitative conclusions to be robust under alternative forms of the interfacial conditions (2.5) (e.g. Halpern \& Gaver 1994; Jensen et al. 2002), and also when $k h_{\mathrm{i}}$ increases towards 1 , even though the results may not be 
accurate quantitatively. The same applies to the lubrication approximation and elasticity models, which also require $k h_{\mathrm{i}} \ll 1$ to be asymptotically accurate.

If the invading fluid is not an inviscid gas, but a second viscous liquid (still having a lower viscosity than the first liquid and immiscible), then steady peeling base states should still exist and, although the linear stability analysis becomes more complicated, our main qualitative conclusions should still hold.

An elastic sheet undergoing peeling is, in general, both under tension and has a bending stiffness. In this case, the peeling solution may be driven predominantly by either tension or bending forces, or by a combination of both (Lister et al. 2013; Peng et al. 2015), but the geometry of the resulting system remains wedge-shaped and hence we can expect the rigid-lid and wedge approximations to apply. Similarly, for systems with different elasticity models (e.g. a thick elastic layer or an elastic half-space), provided that deformations with a given amplitude generate an elastic restoring force that increases with wavenumber and that the base-state solution has a liquid wedge ahead of the interface, we can expect the rigid-lid and wedge approximations to apply.

In the peeling-by-pulling experiments by McEwan \& Taylor (1966), the side edges of the elastic sheet were free to contract in the spanwise direction so that a spanwise tension could not be sustained. This corresponds in the idealized case to the case of nonisotropic tension with tension ratio $\tau=0$ studied in Appendix $\mathrm{C}$, for which we found that a much larger region of parameter space is unstable compared with the isotropic case. In reality the sheet would also have some bending rigidity, which might help to stabilize the system and possibly allow the rigid-lid approximation to apply. If spanwise contraction is prevented, for example by a mechanism keeping the edges of the sheet from moving inward, then the pulling in the longitudinal direction would also generate a spanwise tension. The tension ratio $\tau$ would be equal to the Poisson's ratio of the elastic sheet, which is usually larger than 0.2 , and the rigid-lid approximation applies reasonably well.

Our analysis of steady peeling can also be applied directly to time-varying peeling systems provided that the rate of change of the base state is small compared with the growth rate of the instability, such as in the channel experiments by Ducloué et al. (2017), where the peeling front evolves slowly as it propagates down the channel. Moreover, when the perturbations to the elastic sheet are negligible, the rigid-lid approximation also applies to systems with rapidly varying base states, since the state of the instability is described by the single variable $\hat{R}$. A well-defined time-varying growth rate $\sigma=$ $(\partial \hat{R} / \partial t) / \hat{R}$ can be found using the rigid-lid or wedge approximation, and the linear evolution of the instability is given by $\hat{R}(t)=\hat{R}(0) \exp \left(\int_{0}^{t} \sigma\left(t^{\prime}\right) \mathrm{d} t^{\prime}\right)$. This includes e.g. the case of peeling with a non-wetting liquid that continually accumulates in the wedge rather than being deposited into films coating the surfaces, and any rigid system with a time-evolving converging geometry. A subtle difference for time-dependent base states is that the base velocity gradient $\bar{u}^{\prime}$ can no longer be simplified to $-U h_{0} \alpha_{\mathrm{i}} / h_{\mathrm{i}}^{2}(2.17 b)$. Indeed, it might even be positive (indicating a stretching and hence destabilizing base flow), such as in the case of flow into a stationary converging rigid cell, in which case a long-wavelength instability is expected.

The original motivation for this paper, namely the viscous-fingering instability during injection of air into an elastic-walled Hele-Shaw cell (e.g. Pihler-Puzović et al. 2012), is a complex problem involving a time-dependent base state, a radial geometry and a combination of (non-isotropic) tension and bending forces. The ideas developed here are applied to study it in Pihler-Puzović et al. (2018). 


\section{Acknowledgements}

G.G.P. was supported by an EPSRC PhD studentship. All research data supporting this publication are directly available within this publication.

\section{Appendix A. Numerical method}

\section{A.1. Nondimensionalization of governing equations}

For clarity, we use dimensional variables throughout the main text. However, for the numerical calculations, we use the rescalings

$$
\boldsymbol{x}, R, k^{-1} \sim \mathcal{A} h_{0}, \quad t, \sigma^{-1} \sim \frac{\mathcal{A} h_{0}}{U}, \quad h, h_{\mathrm{i}} \sim h_{0}, \quad p \sim \frac{12 \mu U \mathcal{A}}{h_{0}}, \quad \alpha_{\mathrm{i}} \sim \frac{1}{\mathcal{A}},
$$

which simplify the base-state governing equations $(2.10),(2.12)-(2.14),(2.17)$ to

$$
\begin{gathered}
\bar{p}=-\bar{h}^{\prime \prime} \quad \text { (pulling) } \quad \text { or } \quad \bar{p}=\bar{h}^{\prime \prime \prime \prime} \quad \text { (bending), } \\
\bar{u}=-h^{2} \bar{p}^{\prime}=\frac{\bar{h}-1}{\bar{h}} \text { in } x>0, \quad \bar{h} \rightarrow 1 \text { as } x \rightarrow \infty, \\
\bar{h}=h_{\mathrm{i}}=\frac{1}{f_{1}}, \quad \bar{p}=-\frac{2 f_{2}}{12 C a \mathcal{A} \bar{h}}, \quad \alpha_{\mathrm{i}}=-\bar{h}^{\prime}, \quad \bar{u}^{\prime}=-\frac{\alpha_{\mathrm{i}}}{h_{\mathrm{i}}^{2}} \quad \text { at } x=0^{+},
\end{gathered}
$$

and the perturbation equations $(2.19),(2.24),(2.25)$ to

$$
\begin{aligned}
& \hat{p}=\left(k^{2}-\partial_{x}^{2}\right) \hat{h} \quad \text { (pulling) } \quad \text { or } \quad \hat{p}=\left(k^{2}-\partial_{x}^{2}\right)^{2} \hat{h} \quad \text { (bending), } \\
& \hat{u}=-\bar{h}^{2} \hat{p}^{\prime}-2 \bar{h} \bar{p}^{\prime} \hat{h}, \quad \dot{\hat{h}}=\left(\bar{h}^{3} \hat{p}^{\prime}+3 \bar{h}^{2} \bar{p}^{\prime} \hat{h}+\hat{h}\right)^{\prime}-k^{2} \bar{h}^{3} \hat{p} \quad \text { in } x>0, \\
& \hat{u}+\bar{u}^{\prime} \hat{R}=\left(1-f_{1}-f_{1}^{\prime} C a\right) \dot{\hat{R}} \quad \text { at } x=0^{+}, \\
& \hat{p}+\bar{p}^{\prime} \hat{R}=-\frac{\pi / 4}{12 C a \mathcal{A}^{2}} k^{2} \hat{R}+\frac{2 f_{2}}{12 C a \mathcal{A}}\left(\hat{h}+\bar{h}^{\prime} \hat{R}\right)-\frac{2 f_{2}^{\prime}}{12 \mathcal{A} \bar{h}} \dot{\hat{R}} \quad \text { at } x=0^{+},
\end{aligned}
$$

while (2.22) and (2.23) remain the same.

\section{A.2. Numerical method}

For our numerical calculations, we spatially discretize the equations using a finitedifference scheme on a non-uniform grid which is (iteratively) adapted to ensure that the base state and the perturbations are well resolved (to 1\%). Specifically, the grid is coarser in the interior of the wedge, and then becomes progressively finer both towards the interface $x=0$ and towards the peeling region. Since there is only one spatial dimension, any linear equations involving spatial derivatives give rise to banded matrix equations that are readily solved.

The base-state solutions $(\S 2.2)$ are found using Newton-Raphson iteration starting from the trivial guess $\bar{h}=h_{0}$. For large $C a$, where the solution is $O\left(h_{0}\right)$, the method converges rapidly. For small $C a$, where the final solution is $\bar{h} \gg h_{0}$, it is necessary to solve the system for large $C a$ and then apply numerical continuation to reduce $C a$ to the target value.

To find the dominant mode in $\S 3$ for the perturbation equations, we consider the timedependent form where $\sigma \hat{h}$ in (2.24) and $\sigma \hat{R}$ in (2.25) are replaced by $\partial \hat{h} / \partial t$ and $\partial \hat{R} / \partial t$, respectively, and discretize the system temporally using the implicit backward-Euler method. A naive method to find the dominant mode is to start at an arbitrary initial condition and evolve the system forward until all other modes, which have smaller growth 
rates, have become negligible compared with the dominant one. (Since the equations are linear, the perturbations can be rescaled regularly to keep them at $O(1)$.) We found this method to be viable for large wavenumbers $\left(k \gtrsim k_{\mathrm{vf}}\right)$, and we used it to confirm the accuracy of the following more sophisticated method.

A single time-evolution step is equivalent to multiplying the vector describing the state of the system by a fixed matrix, and the dominant mode corresponds to the eigenvector of the matrix that has maximal eigenvalue. We can thus find it using the Implicitly Restarted Arnoldi Algorithm from the package ARPACK (Lehoucq \& Sorensen 1995). This allowed us to calculate the dominant mode for all values of $k$. Also, once a dominant mode was found, we could use numerical continuation to obtain solutions for slightly different values of $k, C a$ or $\mathcal{A}$. However, we had to go back to the Arnoldi algorithm regularly in order to detect any new modes that might have become dominant instead.

Finally, given the base-state solution, the rigid-lid approximation ( $\S 4$ ) and long-wave expansion (Appendix B) yield banded matrix equations that are straightforward to solve.

\section{Appendix B. Long-wavelength expansion}

We consider the perturbation equations (2.19)-(2.25) in the long-wavelength limit $k \rightarrow 0$, by expanding about the translational mode (3.5) which is the exact solution for $k=0$ and $\sigma=0$. The equations depend on $k$ in three different ways, which we will identify as three different physical mechanisms. In order to keep track of the contributions from each mechanism, we introduce artificial coefficients multiplying each appearance of $k$ in the equations.

In the elasticity equation (2.19), the $O\left(k^{2}\right)$ and $O\left(k^{4}\right)$ terms are due to the spanwise rigidity of the elastic sheet, i.e. its tendency to generate a pressure opposing spanwise variations in the height profile. We multiply the $O\left(k^{2}\right)$ term by $c_{\mathrm{r}}$ and the $O\left(k^{4}\right)$ term, if present, by $c_{\mathrm{r}}^{2}$. As a consequence, each appearance of $k$ in the gas-region solutions $(2.20)$ and the matching conditions $(2.22)$ is multiplied by a factor $\sqrt{c_{\mathrm{r}}}$. In the lubrication equation (2.24), there is a hydrodynamic $O\left(k^{2}\right)$ term which captures how spanwise variations in pressure drive a spanwise flow from regions of high pressure to regions of low pressure. At leading order, the regions of high pressure are the locations where the peeling has advanced ahead of its mean position, so the effect of this term is analogous to the viscous driving force in the classical viscous-fingering instability. We multiply this term by the coefficient $c_{\mathrm{v}}$. Finally, in the dynamic interface equation $(2.25 a)$, the $O\left(k^{2}\right)$ term represents horizontal surface tension, and we multiply it by $c_{\gamma}$.

Hence, the artificially modified equations for the liquid region depend on $k$ via the quantities $\sqrt{c_{\mathrm{r}}} k, c_{\mathrm{v}} k^{2}$, and $c_{\gamma} k^{2}$, and we make the ansatz

$$
\begin{gathered}
\sigma=\sigma_{0}+k\left(\sqrt{c_{\mathrm{r}}} \sigma_{1 \mathrm{r}}\right)+k^{2}\left(c_{\mathrm{r}} \sigma_{2 \mathrm{r}}+c_{\mathrm{v}} \sigma_{2 \mathrm{v}}+c_{\gamma} \sigma_{2 \gamma}\right)+O\left(k^{3}\right), \\
\frac{\hat{h}}{\hat{R}}=\hat{h}_{0}+k\left(\sqrt{c_{\mathrm{r}}} \hat{h}_{1 \mathrm{r}}\right)+k^{2}\left(c_{\mathrm{r}} \hat{h}_{2 \mathrm{r}}+c_{\mathrm{v}} \hat{h}_{2 \mathrm{v}}+c_{\gamma} \hat{h}_{2 \gamma}\right)+O\left(k^{3}\right) .
\end{gathered}
$$

(The other variables $\hat{p}$ and $\hat{u}$ are expanded similarly, and are treated in parallel with $\hat{h}$. Henceforth, for brevity, we only mention $\hat{h}$ explicitly.) The unmodified equations are recovered by setting $c_{\mathrm{r}}=c_{\mathrm{v}}=c_{\gamma}=1$.

At $O\left(k^{0}\right)$ we recover the equations for the translating mode, which are satisfied by $\sigma_{0}=0$ and $\hat{h}_{0}=-\bar{h}^{\prime}$.

At $O\left(k^{1}\right)$, the equations for $\sigma_{1 \mathrm{r}}$ and $\hat{h}_{1 \mathrm{r}}$ are completely homogeneous except for the 

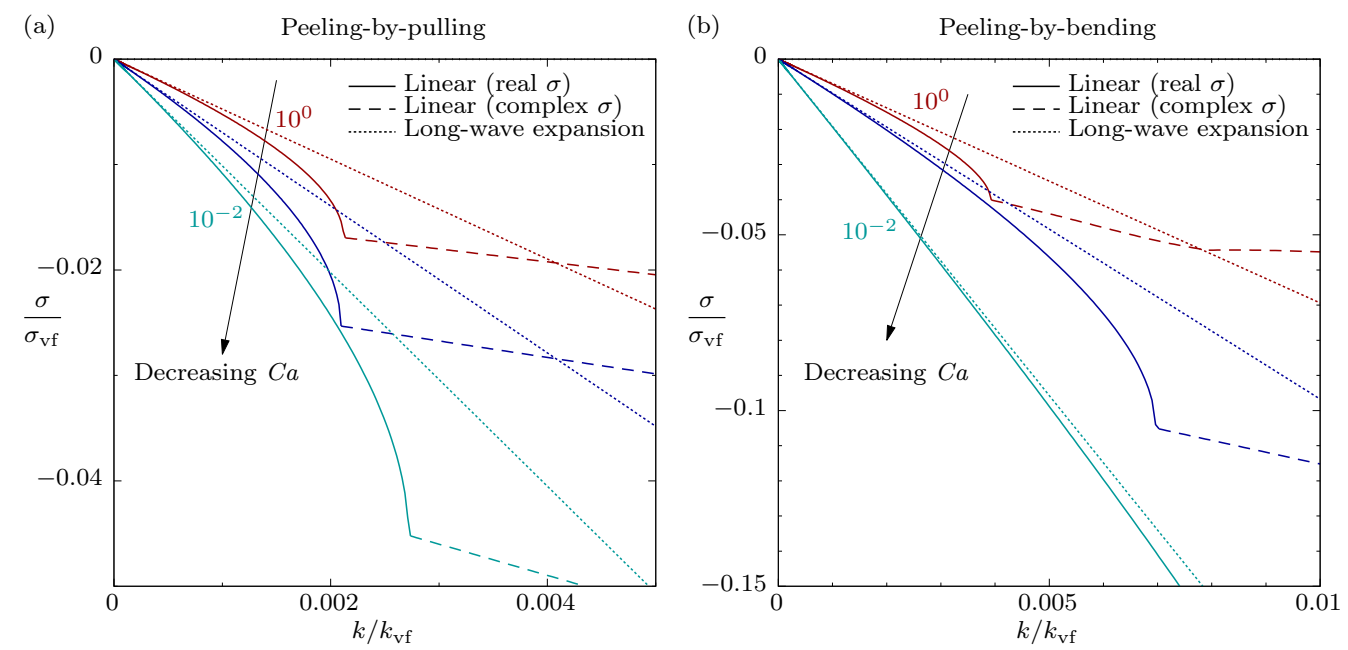

FiguRE 12. Growth rate $\sigma$ of the dominant mode for each wavenumber $k$, for $\mathcal{A}=10$ and $C a=10^{0}, 10^{-1}, 10^{-2}$, rescaled using the viscous-fingering scales (3.4), comparing results from the full linear calculation $(\S 3)$ and the leading-order term of the long-wave expansion (B 1a). For the dashed parts of the curves, the dominant growth rate is complex and only the real part is plotted.

boundary matching condition (2.22), which contains forcing terms as follows

$$
\begin{array}{lll}
\hat{h}_{1 \mathrm{r}}^{\prime}-\hat{h}_{0}=0 & \text { at } x=0^{+} & \text {(pulling), } \\
\hat{h}_{1 \mathrm{r}}^{\prime \prime}-2 \hat{h}_{0}^{\prime}=\hat{h}_{1 \mathrm{r}}^{\prime \prime \prime}-2 \hat{h}_{0}^{\prime \prime}=0 & \text { at } x=0^{+} & \text {(bending). }
\end{array}
$$

We evaluate these conditions as

$$
\begin{aligned}
-\hat{h}_{1 \mathrm{r}}^{\prime}(0) & =-\hat{h}_{0}(0)=\bar{h}^{\prime}(0) \quad<0 & & \text { (pulling) } \\
\hat{h}_{1 \mathrm{r}}^{\prime \prime}(0) & =2 \hat{h}_{0}^{\prime}(0)=-2 \bar{h}^{\prime \prime}(0)<0 & & \text { (bending) } .
\end{aligned}
$$

(The second condition for peeling-by-bending yields $\hat{h}_{1 \mathrm{r}}^{\prime \prime \prime}(0)=2 \hat{h}_{0}^{\prime \prime}(0)=2 \bar{h}^{\prime \prime \prime}(0)=0$ which is unimportant.) The slope $-h^{\prime}(0)$ and curvature $h^{\prime \prime}(0)$ drive the peeling-by-pulling and peeling-by-bending processes, respectively, and larger values of these peeling quantities result in larger peeling speeds (Lister et al. 2013; Peng et al. 2015). Hence, the negative signs in (B 3) show that the locations where the perturbed interface is ahead experience an $O\left(\sqrt{c_{\mathrm{r}}} k\right)$ reduction in the peeling quantity, thus slowing down the peeling, while locations where the interface is lagging behind speed up. This is a stabilizing effect, and indeed our numerical solution of the $O\left(k^{1}\right)$ equations yield $\sigma_{1 \mathrm{r}}<0$ throughout the parameter space.

At $O\left(k^{2}\right)$, the forcing terms are given by the viscous $O\left(c_{\mathrm{v}} k^{2}\right)$ term in the lubrication equation (2.24), the capillary $O\left(c_{\gamma} k^{2}\right)$ term in the dynamic interface equation $(2.25 b)$, and various $O\left(c_{\mathrm{r}} k^{2}\right)$ terms in (2.19) and (2.22) as well as a term proportional to $\sigma_{1 \mathrm{r}} \hat{h}_{1 \mathrm{r}}$ in (2.24). Numerical solutions of the relevant equations show that, for all parameter values, the rigidity and capillary term are stabilizing $\left(\sigma_{2 \mathrm{r}}, \sigma_{2 \mathrm{v}}<0\right)$ while the viscous term is destabilizing $\left(\sigma_{2 \mathrm{v}}>0\right)$.

Figure 13 shows magnifications of the region around $k=0$ from figure 4 with the leading-order result $\sigma \approx k \sigma_{1 \mathrm{r}}$ (B $1 a$ ) from the long-wavelength expansion overlaid (with $\left.c_{\mathrm{r}}=1\right)$. There is an excellent agreement for small values of $k$, and, as $k$ increases, the 
numerical results begin to curve away from the linear result as expected, before having a sharp transition to a different mode (which is oscillatory).

\section{Appendix C. Non-isotropic tension}

While bending stiffness is an intrinsic property of the elastic sheet and does not depend on which direction the sheet is being bent in, the tension in a sheet depends on the boundary conditions applied, and hence need not be isotropic. Indeed, in realistic situations the mechanism that supplies the longitudinal tension $T$ (in the $x$-direction), which drives the peeling, may be different from the mechanism that supplies the spanwise tension (in the $y$-direction), which helps to prevent corrugations forming in the sheet. In this appendix, we relax the assumption that the tension is isotropic and denote the spanwise tension by $\tau T$, where the tension ratio $\tau \geqslant 0$.

The peeling base state has no spanwise variation, so it is the same as described in $\S 2.2$. For the linear perturbations, we have analysed the isotropic case $\tau=1$ in detail in the main part of the paper, and shown in $\S 4$ that for this case the spanwise tension is sufficiently strong to keep the sheet approximately rigid. Hence, the rigid-lid approximation is expected to apply for general values of $\tau \geqslant 1$, and in this section we focus on values $0 \leqslant \tau<1$, when the perturbations to the elastic sheet may have a significant effect on the perturbation flow and instability.

\section{C.1. Governing equations}

The elasticity law (2.1) and its linearized version (2.19) are modified to

$$
p=-T\left(\partial_{x}^{2}+\tau \partial_{y}^{2}\right) h \quad \text { and } \quad \hat{p}=T\left(\tau k^{2}-\partial_{x}^{2}\right) \hat{h},
$$

and as stated above the base-state equations and solutions remain unchanged. The new elasticity equation (C 1) changes the gas-region solution (2.20) and hence the resulting boundary condition $(2.22 a)$ for the liquid region to

$$
\hat{h}=A_{4} e^{\sqrt{\tau} k x} \quad \text { in } x<0 \quad \text { and } \quad \hat{h}^{\prime}-\sqrt{\tau} k \hat{h}+\bar{h}^{\prime \prime} \hat{R}=0 \quad \text { at } x=0^{+} .
$$

The remaining perturbation equations (2.23)-(2.25b) are unchanged.

\section{C.2. Numerical results}

We solve the new perturbation equations (C 1), (C 2), (2.23)-(2.25b) using the same method as described in $\S 3.2$ and Appendix A.

Figure 13(a) shows the rescaled growth rate, analogous to figures 4 and 7, for various values of $\tau$, including the isotropic case $\tau=1$ for comparison. We find that a smaller value of $\tau$ (i.e. the sheet being more flexible) typically leads to a larger value of $\sigma$ (i.e. a stronger instability), which is in agreement with our understanding of rigidity as a stabilizing effect. The change is fairly small as $\tau$ is reduced from 1 to 0.1 , indicating that the weaker spanwise tension is still sufficient to keep the elastic sheet effectively rigid. However, for $\tau=0$, while there is not much of a change for $C a=1$, the difference becomes significant for smaller values of $C a$. In particular, for $C a=0.01$ there is a stark contrast between the cases $\tau \geqslant 0.1$ for which the system is stable $(\sigma \leqslant 0$ for all $k$ ), and the case $\tau=0$ for which the system is unstable $(\sigma>0$ for a range of $k$ ).

The long-wavelength expansion in Appendix B can be adapted to the case of nonisotropic tension simply by interpreting the artificial coefficient $c_{\mathrm{r}}$ as the tension ratio $\tau$, while retaining the values $c_{\mathrm{v}}=c_{\gamma}=1$ for the other two coefficients. Hence, for $\tau>0$, 

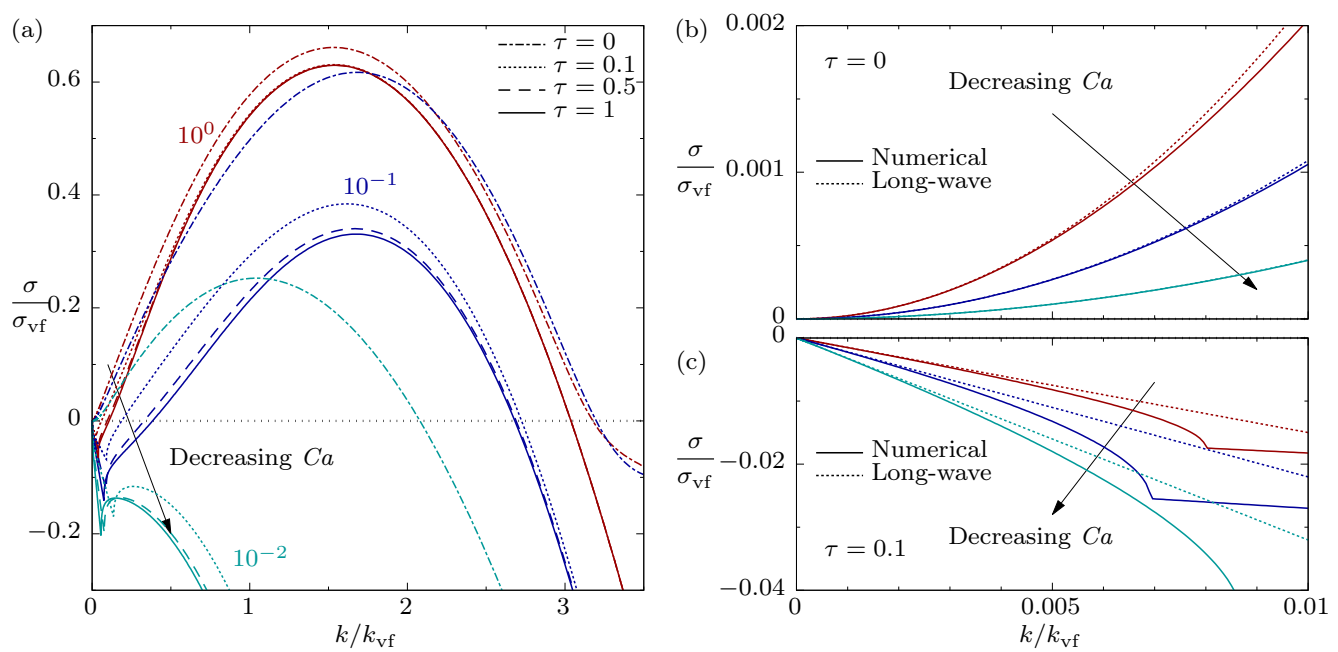

Figure 13. Growth rate $\sigma$ of the dominant mode for each wavenumber $k$, for $\mathcal{A}=10$, $C a=10^{0}, 10^{-1}, 10^{-2}$, and $\tau=0,0.1,0.5,1$, rescaled using the viscous-fingering scales $(3.4 a)$. (Cf. figures 4 and 7 .)

we still have the leading-order linear behaviour $\sigma \approx \sqrt{\tau} \sigma_{1 \mathrm{r}}<0$, as shown in figure 13(c) which shows a magnification of the region near $k=0$ of figure 13(a) for $\tau=0.1$.

However, for $\tau=0$ (figure 13b), the $O(k)$ term vanishes and we are left with a quadratic leading-order term $\sigma \approx\left(\sigma_{2 \mathrm{v}}+\sigma_{2 \gamma}\right) k^{2}$ in the long-wavelength expansion, whose sign is determined by a competition between the destabilizing viscous coefficient $\sigma_{2 \mathrm{v}}>0$ and the stabilizing capillary coefficient $\sigma_{2 \gamma}<0$. For the parameter values used in this figure, $\sigma_{2 \mathrm{v}}+\sigma_{2 \gamma}>0$. Hence the long-wavelength mode, and consequently the entire system, is unstable. In general, for $\tau=0$, the condition $\sigma_{2 \mathrm{v}}+\sigma_{2 \gamma}>0$ is a sufficient condition for the system to be unstable.

Figure 14 shows the contours of the rescaled dominant growth rate $\sigma / \sigma_{\mathrm{vf}}$ in the full parameter space for various values of $\tau$, with the results for $\tau=1$ (figure $5 a$ ) overlaid for comparison. In all cases, we find that the behaviour of the dominant mode is unchanged when the instability is strong $\left(\sigma \gtrsim \sigma_{\mathrm{vf}}\right)$ but that the reduction in $\tau$ makes the system more unstable when the instability is weaker. This is consistent with our observations in figure $13(a)$. For $\tau=0.1$ the region of stability is only slightly reduced compared with $\tau=1$, but as $\tau$ decreases the region shrinks further until it almost disappears completely for $\tau=0$.

For $\tau=0$, our numerical results indicate that if $\sigma_{2 \mathrm{v}}+\sigma_{2 \gamma}<0$, then $\sigma$ is negative not only for small $k>0$ but also for all larger values of $k$. In other words, the stability boundary in figure $14(c)$ coincides with the curve $\sigma_{2 \mathrm{v}}+\sigma_{2 \gamma}=0$.

\section{Appendix D. Scaling arguments in the rigid-lid approximation}

\section{D.1. Justification of simplification of equations}

We use scaling estimates to justify the neglect of all instances of $\hat{h}$ in the perturbation equations (2.24) and (2.25) in the large-wavenumber limit $K=k h_{\mathrm{i}} / \alpha_{\mathrm{i}} \gg 1$, in order to arrive at the rigid-lid approximation discussed in $\S 4$. The main assumptions are that the longitudinal length scale of the perturbations is equal to the spanwise length scale $1 / k$, while the longitudinal length scale of the base-state solution is $L_{\mathrm{w}} \sim h_{\mathrm{i}} / \alpha_{\mathrm{i}} \gg 1 / k$. 

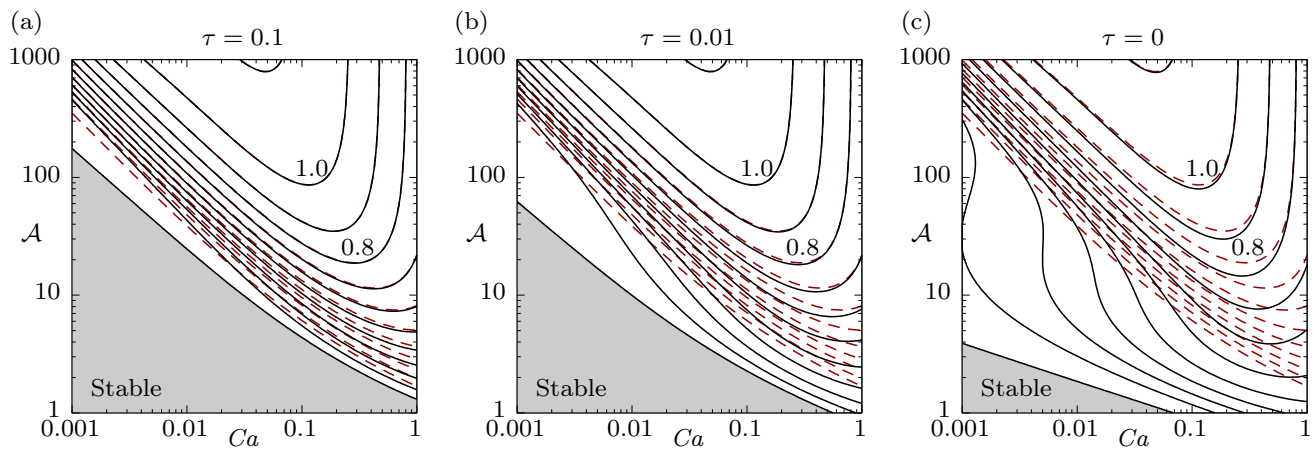

Figure 14. Contours of the rescaled maximal growth rate $\sigma_{\mathrm{m}} / \sigma_{\mathrm{vf}}=\sigma_{\mathrm{m}} /\left(U \sqrt{C a} / h_{\mathrm{i}}\right)$ as a function of the parameters $C a$ and $\mathcal{A}$ for three values of the tension ratio $\tau$ (solid curves), and the isotropic case $\tau=1$ (dashed curves) from figure 5(a) for comparison. The contour spacing is 0.1 , and the labels indicate some of the contour values.

In the lubrication equation (2.24b), we use the viscous-fingering scaling $\sigma \sim k U$ and find that the terms involving $\hat{h}$ on both the left-hand side and the right-hand side scale like $k U \hat{h}$, while the terms involving $\hat{p}$ scale like $k^{2} \bar{h}^{3} \hat{p} / 12 \mu$. The ratio between these can be simplified using the scaling $U \sim \bar{h}^{2} \bar{p} /\left(12 \mu L_{w}\right)(2.13)$ as

$$
\frac{k U \hat{h}}{k^{2} \bar{h}^{3} \hat{p} /(12 \mu)} \sim \frac{1}{k L_{\mathrm{w}}} \frac{\bar{p} / \bar{h}}{\hat{p} / \hat{h}} \sim \begin{cases}\left(k L_{\mathrm{w}}\right)^{-3} \ll 1 & \text { (pulling), } \\ \left(k L_{\mathrm{w}}\right)^{-5} \ll 1 & \text { (bending). }\end{cases}
$$

Hence the terms with $\hat{h}$ can be dropped to yield $(4.2 b)$, and a similar argument applies for $(4.2 a)$.

In the dynamic interfacial condition (2.25b), we compare the terms inside the parentheses representing vertical surface tension. Their ratio can be estimated, using (2.13) again and $\hat{R} \sim \bar{h}^{2} \hat{p} /(12 \mu U)(3.4)$, as

$$
\frac{\hat{h}}{\bar{h}^{\prime} \hat{R}} \sim \frac{\hat{h} / \hat{p}}{\bar{h} / \bar{p}} \sim \begin{cases}\left(k L_{\mathrm{w}}\right)^{-2} \ll 1 & \text { (pulling) }, \\ \left(k L_{\mathrm{w}}\right)^{-4} \ll 1 & \text { (bending) }\end{cases}
$$

so again $\hat{h}$ can be neglected.

Hence, the rigid-lid approximation holds when $K \sim k L_{\mathrm{w}} \gg 1$.

\section{D.2. Analysis of the limit $C a \ll 1$}

We calculate scaling estimates for the maximal growth rate $\sigma_{\mathrm{m}}$ and its associated wavenumber $k_{\mathrm{m}}$ in the unstable region for $C a \ll 1$, under the assumption $K=k h_{\mathrm{i}} / \alpha_{\mathrm{i}} \gg$ 1 which is justified a posteriori.

Since $C a \ll 1, K \gg 1$, and $\alpha_{\mathrm{i}} \lesssim C a(4.10)$, the admittance and the denominator of the growth-rate expression (4.8) are both approximately equal to 1 , so we can maximize $\sigma+\left(-\bar{u}^{\prime}\right)$ over $k$ to find scaling estimates

$$
k_{\mathrm{m}} \sim \frac{S C a^{1 / 2}}{h_{\mathrm{i}}}=S k_{\mathrm{vf}}, \quad \sigma_{\mathrm{m}}+\left(-\bar{u}^{\prime}\right) \sim \frac{U}{h_{\mathrm{i}}} S^{3} C a^{1 / 2}=S^{3} \sigma_{\mathrm{vf}},
$$

where $S^{2}=1-f_{1}-2 f_{2} \alpha_{\mathrm{i}} /(12 C a)$ represents the viscous driving force with the $k$ independent stabilizing terms subtracted and the classical viscous-fingering scales $k_{\mathrm{vf}}$ and $\sigma_{\mathrm{vf}}$ are given by (3.4). Away from the stability boundary, we have $S=O(1)$ and recover the classical viscous-fingering scale. As the stability boundary is approached, $S$ 
decreases, and at marginal stability $\alpha_{\mathrm{i}} \approx 6 C a(4.10)$ we obtain

$$
S \sim C a^{7 / 18}, \quad k_{\mathrm{m}} \sim C a^{7 / 18} k_{\mathrm{vf}},
$$

by setting $\sigma_{\mathrm{m}}=0$ in (D $\left.3 b\right)$ and using (2.17).

We can thus estimate, using $\alpha_{\mathrm{i}} \leqslant 6 C a$,

$$
K=\frac{k_{\mathrm{m}} h_{\mathrm{i}}}{\alpha_{\mathrm{i}}} \sim \frac{S C a^{1 / 2}}{\alpha_{\mathrm{i}}} \gtrsim \frac{S}{C a^{1 / 2}} .
$$

Away from the stability boundary we have $K \sim C a^{-1 / 2} \gg 1$, but as the boundary is approached $S$ decreases until $K \sim C a^{-1 / 9}$ is achieved at marginal stability. Hence, although $K \rightarrow \infty$ as $C a \rightarrow 0$, a very small value of $C a$ is necessary to yield a reasonably large value of $K$. Thus, we have justified the use of the limit $K \gg 1$ which was necessary to derive the rigid-lid approximation and make the problem analytically tractable, and at the same time explained why the magnitude of $K$ is only assumed to satisfy $K \gtrsim 1$, rather than $K \gg 1$, in some parts of the calculation.

Finally, we use the result (D $4 b$ ) to find that the four terms contributing to stability in (4.9) are of order

$$
O\left(C a^{2 / 3}\right), \quad O(1), \quad O\left(C a^{7 / 9}\right), \quad O\left(C a^{7 / 9}\right),
$$

respectively, which confirms that the terms representing films, horizontal surface tension and kinematic compression yield small corrections to the stability criterion (4.10) based on taper alone.

\section{Appendix E. Parabolic wedge approximation for peeling-by-bending}

In order to explain the small discrepancy in the admittance between the $\mathrm{Ca} \rightarrow 0$ limiting behaviour of the rigid-lid approximation and the wedge approximation in figure $8(b)$, we revisit the peeling-by-bending base-state (figure 2) and note that the shape of the liquid wedge is closer to parabolic than triangular. This fact is confirmed by the theoretical analyses of Lister et al. (2013) and Peng et al. (2015). Hence, we approximate the wedge as having a parabolic shape, with the given height $h_{\mathrm{i}}$ and slope $\alpha_{\mathrm{i}}$ at the interface,

$$
\bar{h}=\frac{\alpha_{\mathrm{i}}^{2}}{4 h_{\mathrm{i}}} s^{2}, \quad \text { where } s=\frac{2 h_{\mathrm{i}}}{\alpha_{\mathrm{i}}}-x,
$$

which can also be considered a second-order Taylor expansion of the height profile. Solving (4.2) yields the perturbation pressure profile

$$
\hat{p} \propto\left(\frac{3}{(k s)^{5}}-\frac{3}{(k s)^{4}}+\frac{1}{(k s)^{3}}\right) e^{k s}-\left(\frac{3}{(k s)^{5}}+\frac{3}{(k s)^{4}}+\frac{1}{(k s)^{3}}\right) e^{-k s},
$$

and hence the admittance

$$
Y_{p}(K)=\frac{1-\frac{3}{K}+\frac{15}{4 K^{2}}-\frac{15}{8 K^{3}}+e^{-4 K}\left(1+\frac{3}{K}+\frac{15}{4 K^{2}}+\frac{15}{8 K^{3}}\right)}{1-\frac{3}{2 K}+\frac{3}{4 K^{2}}-e^{-4 K}\left(1+\frac{3}{2 K}+\frac{3}{4 K^{2}}\right)}, \quad \text { where } \quad K=\frac{k h_{\mathrm{i}}}{\alpha_{\mathrm{i}}} .
$$

This result is plotted as the dot-dashed curve in figure $8(b)$, and is indeed the curve towards which the rigid-lid results are converging in the limit $C a \rightarrow 0$. We have compared the full spectrum with the results in figure $7(\mathrm{~b})$, and found that it is nearly indistinguishable from the rigid-lid results. 


\section{REFERENCES}

Al-Housseiny, T. T., Christov, I. C. \& Stone, H. A. 2013 Two-phase fluid displacement and interfacial instabilities under elastic membranes. Phys. Rev. Lett. 111, 034502.

Al-Housseiny, T. T., Tsai, P. A. \& Stone, H. A. 2012 Control of interfacial instabilities using flow geometry. Nat. Phys. 8 (10), 747-750.

Balmforth, N. J., Craster, R. V. \& Hewitt, I. J. 2014 The speed of an inclined ruck. Proc. R. Soc. A 471, 20140740.

Bretherton, F. P. 1961 The motion of long bubbles in tubes. J. Fluid Mech. 10 (2), 166-188.

Chuoke, R. L., Van Meurs, P. \& van der Poel, C. 1959 The instability of slow, immiscible, viscous liquid-liquid displacements in permeable media. T. Am. I. Min. Met. Eng. 216, $188-194$.

Ducloué, L., Hazel, A. L., Pihler-Puzović, D. \& Juel, A. 2017 Viscous fingering and dendritic growth under an elastic membrane. J. Fluid Mech. 826, R2.

Grenfell-Shaw, J. C. \& Woods, A. W. 2017 The instability of a moving interface in a narrow tapering channel of finite length. J. Fluid Mech. 831, 252-270.

Halpern, D. \& Gaver, D. P. 1994 Boundary element analysis of the time-dependent motion of a semi-infinite bubble in a channel. J. Comput. Phys. 115 (2), 366-375.

Hewitt, I. J., Balmforth, N. J. \& De Bruyn, J. R. 2015 Elastic-plated gravity currents. Eur. J. Appl. Math. 26 (1), 1-31.

HiLl, S. 1952 Channelling in packed columns. Chem. Eng. Sci. 1 (6), 247-253.

Homsy, G. M. 1987 Viscous fingering in porous media. Annu. Rev. Fluid Mech. 19, 271-311.

Hosoi, A. E. \& Mahadevan, L. 2004 Peeling, healing, and bursting in a lubricated elastic sheet. Phys. Rev. Lett. 93, 137802.

Jensen, O. E., Horsburgh, M. K., Halpern, D. \& Gaver, D. P. 2002 The steady propagation of a bubble in a flexible-walled channel: Asymptotic and computational models. Phys. Fluids 14 (2), 431-442.

Lehoucq, R. B. \& Sorensen, D. C. 1995 Deflation Techniques for an Implicitly Restarted Arnoldi Iteration. SIAM. J. Matrix Anal. 83 Appl. 17, 789-821.

Lister, J. R., Peng, G. G. \& Neufeld, J. A. 2013 Viscous control of peeling an elastic sheet by bending and pulling. Phys. Rev. Lett. 111, 154501.

McEwan, A. D. \& TAYlor, G. I. 1966 The peeling of a flexible strip attached by a viscous adhesive. J. Fluid Mech. 26 (1), 1-15.

Pearson, J. R. A. 1960 The instability of uniform viscous flow under rollers and spreaders. $J$. Fluid Mech. 7 (4), 481-500.

Peng, G. G., Pihler-Puzović, D., Juel, A., Heil, M. \& Lister, J. R. 2015 Displacement flows under elastic membranes. Part 2: Analysis of interfacial effects. J. Fluid Mech. 784, $512-547$.

Pihler-Puzović, D., Illien, P., Heil, M. \& Juel, A. 2012 Suppression of complex fingerlike patterns at the interface between air and a viscous fluid by elastic membranes. Phys. Rev. Lett. 108, 074502.

Pinler-Puzović, D., Juel, A. \& Heil, M. 2014 The interaction between viscous fingering and wrinkling in elastic-walled Hele-Shaw cells. Phys. Fluids 26, 1-24.

Pihler-Puzović, D., Juel, A., Peng, G. G., Lister, J. R. \& Heil, M. 2015 Displacement flows under elastic membrances. Part 1: Experiments and direct numerical simulations. J. Fluid Mech. 784, 487-511.

Pihler-Puzović, D., Peng, G. G., Lister, J. R., Heil, M. \& Juel, A. 2018 Viscous fingering in a radial elastic-walled Hele-Shaw cell. J. Fluid Mech. (submitted) .

Pihler-Puzović, D., Périllat, R., Russell, M., Juel, A. \& Heil, M. 2013 Modelling the suppression of viscous fingering in elastic-walled Hele-Shaw cells. J. Fluid Mech. 731, $162-183$.

Reinelt, D. A. \& Saffman, P. G. 1985 The penetration of a finger into a viscous fluid in a channel and tube. SIAM J. Sci. Stat. Comp. 6 (3), 542-561.

REynolds, O. 1886 Iv. on the theory of lubrication and its application to mr. beauchamp tower's experiments, including an experimental determination of the viscosity of olive oil. Phil. Trans. R. Soc. Lond. 177, 157-234.

Ruschak, K. J. 1985 Coating flows. Annu. Rev. Fluid Mech. 17 (1), 65-89. 
SAFFman, P. G. \& TAYlor, G. I. 1958 The penetration of a fluid into a porous medium or Hele-Shaw cell containing a more viscous liquid. Proc. R. Soc. London Ser. A 245 (1242), $312-329$. 